\title{
Robust unsupervised small area change detection from SAR imagery using deep learning
} \author{
Xinzheng Zhang ${ }^{\mathrm{a}, \mathrm{b}, *}$, Hang $\mathrm{Su}^{\mathrm{a}}$, Ce Zhang ${ }^{\mathrm{c}, \mathrm{d},{ }^{*}, \text { Xiaowei Gu}}{ }^{\mathrm{e}}$, Xiaoheng Tan ${ }^{\mathrm{a}, \mathrm{b}}$, Peter M. Atkinson ${ }^{\mathrm{c}, \mathrm{f}, \mathrm{g}}$ \\ ${ }^{a}$ School of Microelectronics and Communication Engineering, Chongqing University, Chongqing, 400044, China \\ ${ }^{b}$ Chongqing Key Laboratory of Space Information Network and Intelligent Information Fusion, Chongqing, 400044, China \\ ${ }^{c}$ Lancaster Environment Centre, Lancaster University, Lancaster, LA1 4YQ, United Kingdom \\ ${ }^{d} U K$ Centre for Ecology \& Hydrology, Library Avenue, Lancaster, LA1 4AP, United Kingdom \\ e Department of Computer Science, Aberystwyth University, Aberystwyth, SY23 3DB, United Kingdom \\ ${ }^{f}$ Geography and Environmental Science, University of Southampton, Highfield, Southampton SO17 1BJ, UK \\ g Institute of Geographic Sciences and Natural Resources Research, Chinese Academy of Sciences, 11A Datun Road, Beijing \\ 100101, China
}

\section{Abstract}

Small area change detection using synthetic aperture radar (SAR) imagery is a highly challenging task, due to speckle noise and imbalance between classes (changed and unchanged). In this paper, a robust unsupervised approach is proposed for small area change detection using deep learning techniques. First, a multi-scale superpixel reconstruction method is developed to generate a difference image (DI), which can suppress the speckle noise effectively and enhance edges by exploiting local, spatially homogeneous information. Second, a two-stage centre-constrained fuzzy c-means clustering algorithm is proposed to divide the pixels of the DI into changed, unchanged and intermediate classes with a parallel clustering strategy. Image patches belonging to the first two classes are then constructed as pseudo-label training samples, and image patches of the intermediate class are treated as testing samples. Finally, a convolutional wavelet neural network $(\mathrm{CWNN})$ is designed and trained to classify testing samples into changed or unchanged classes, coupled with a deep convolutional generative adversarial network (DCGAN) to increase the number of changed class within the pseudo-label training samples. Numerical experiments on four real SAR datasets demonstrate the validity and robustness of the proposed approach, achieving up to $99.61 \%$ accuracy for small area change detection.

\section{Keywords:}

Change detection; Synthetic aperture radar; Difference image; Fuzzy c-means algorithm; Deep learning. 


\section{Introduction}

Remotely sensed change detection focuses on identifying land-cover changes by analysing the images observed over the same scene at different times (Huang et al., 2011; Li et al., 2020; Cao et al., 2020). In particular, remotely sensed synthetic aperture radar (SAR) imagery is adopted widely for change detection, owing to its ability to penetrate cloud cover, and its insensitivity to atmospheric and lighting conditions (Wang et al., 2016; Gong et al., 2017; Li et al., 2019). Change detection using SAR imagery has been applied in a variety of real-world settings, such as for earthquake damage assessment (Brunner et al., 2010), forest mapping (Pantze et al., 2014) and flood monitoring (Kim et al., 2020). Over the past decades, tremendous effort has been made to develop automatic change detection methods using multi-temporal SAR images. Amongst them, machine learning is currently considered as the most promising and evolving set of approaches (Gong et al., 2017). In general, machine learning-based change detection can be divided into supervised and unsupervised approaches (Li et al., 2019; Geng et al., 2019). The major issue in relation to the supervised approach is the lack of ground reference data, and it often involves manual labelling processes that are labour-intensive and time-consuming (Saha et al., 2020). Thus, unsupervised approaches are developed and employed widely in this field (Li et al., 2015; Jia et al., 2016). The major components of unsupervised approaches include: 1) image pre-processing, 2) difference image (DI) generation, and 3) analysis of the DI and the classification of pixels into changed and unchanged classes (Li et al., 2020; Wang et al., 2020).

The image pre-processing step is used to co-register the SAR images (Lei et al., 2019) and filter speckle noise (Gong et al., 2012; Gong et al., 2016; Li et al., 2018). In SAR images, speckle noise severely degrades the quality of images, thus affecting the performance of subsequent tasks. Numerous methods have been developed to remove speckle noise from SAR images, such as Frost filtering (Sun et al., 2020), non-local means approach (Deledalle et al., 2015), and deep learning method (Cozzolino et al., 2020).

DI generation is aim to provide guidance for post-processing. Subtraction (S) and logarithmic ratio (LR) operators are two basic techniques to obtain pixel-by-pixel DI. Some approaches exploited spatial information in a local window for DI generation, such as the mean ratio (MR) and the neighbourhood-based ratio (NR) (Inglada et al., 2007; Gong et al., 2012), where speckle noise is reduced and the discrimination capability of DI is enhanced. 
The DI analysis is a critical step, by which change detection is converted into a binary classification task

59 (e.g. Thresholding or $k$-means clustering). Typically, fuzzy $c$-means (FCM) is a widely adopted clustering 60 approach in change detection using SAR imagery (Gong et al., 2012; Gao et al., 2019; Li et al., 2019). 61 Several FCM variants have been proposed to reduce the speckle noise while retaining spatial details (Krinidis et al., 2010; Gong et al., 2012; Tian et al., 2018). Recent researches suggest that the DI should be divided into three categories: high-probability changed, high-probability unchanged, and an intermediate class (Li et al., 2019; Kalaiselvi et al., 2020). The intermediate class represents pixels that are difficult to discriminate by a specific clustering algorithm, which are generally differentiated by a deep learning classifier such as a convolutional neural network $(\mathrm{CNN})$ or a convolutional-wavelet neural network (CWNN) (Li et al. 2019; Gao et al., 2019).

One common issue in unsupervised deep learning approaches is to deal with small area changes from multi-temporal SAR images. Such a problem exists in multiple steps as follows:

DI generation: most DI generation methods adopt rectangular windows to characterise local spatial information. Such strategy will smooth out small area changes or fine details along the changed and unchanged region, where changed pixels are difficult to identify (Wang et al., 2020).

The clustering-based DI analysis: many FCM-based methods fail to produce accurate results, due mainly to the use of overall optimisation objective, which forces the cluster prototype of the minority (changed) class to migrate to the majority (unchanged) class, particularly for the imbalanced data distribution.

Training a deep learning classifier: The number of changed pixels is far less than unchanged pixels in small area change detection, where the pseudo-label training samples of the changed class are insufficient to support the training of deep learning classifier, leading to poor classification accuracy.

To address these issues, we developed a novel set of methods as Robust Unsupervised Small Area Change Detection (RUSACD) approach. First, a novel DI generation method is proposed based on multiscale superpixel reconstruction (MSRDI), by exploiting the local spatial information within a superpixel (i.e., an image object) instead of a regular window. Multi-scale information is extracted in superpixel segmentation and DI reconstruction, followed by fusion to achieve the final MSRDI. Second, a two-stage centre-constrained FCM algorithm (TCCFCM) is developed to group imbalanced data. The TCCFCM is designed to identify the preliminary and reliable clustering centres of the changed and unchanged classes. 
of cluster prototypes. Finally, a deep convolutional generative adversarial network (DCGAN) was embedded in this framework for data augmentation, which addressed the issue of insufficient training samples and class 89 imbalance.

90 The major contributions include:1) a novel MSRDI generating method was developed, which can 91 effectively enhance the quality and separability of the DI. 2) TCCFCM was proposed for analysing MSRDI, coupled with a parallel clustering strategy to divide the pixels of MSRDI into three classes: changed, unchanged and intermediate classes. 3) a DCGAN was applied to increase the number of training samples and the visual features of the changed class and, thus, achieve a balance of training samples amongst classes. This was followed by a CWNN to differentiate the intermediate class into changed and unchanged classes. The new method was tested comprehensively on six real SAR image datasets. The overall pipeline of the

RUSACD is shown in Fig. 1, demonstrating how each component is linked together in a modular design.

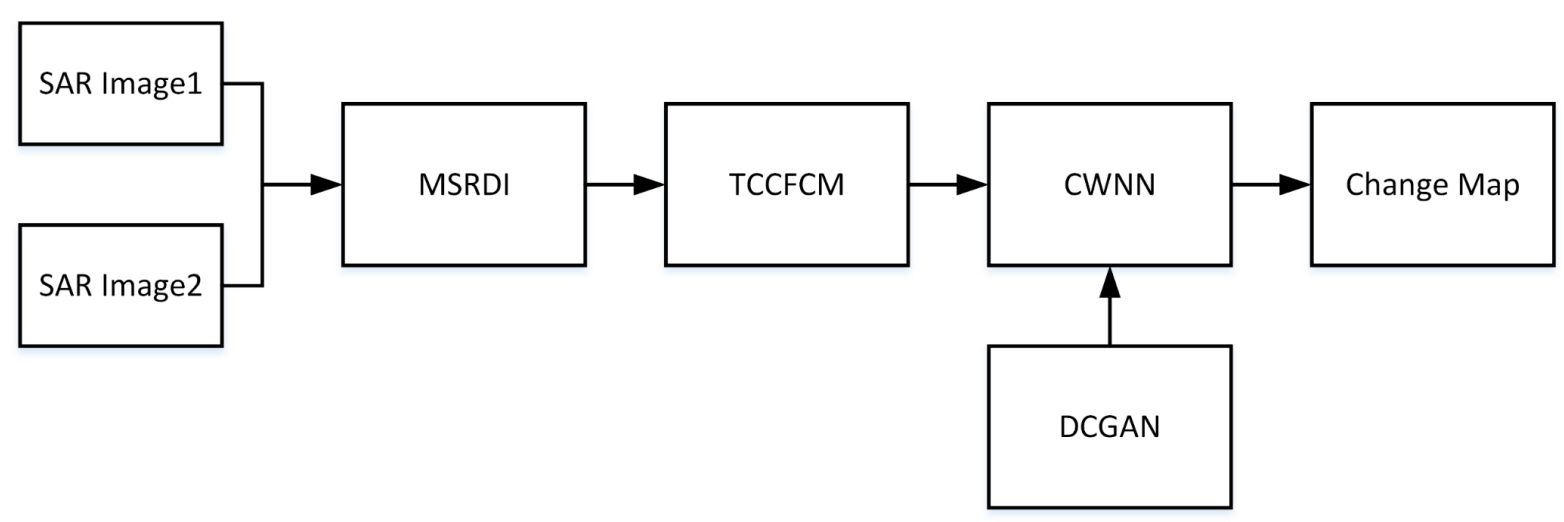

Fig.1 Simple flow diagram illustrating the processing pipeline.

\section{Related work}

\subsection{DI generation}

In the step of DI generation, several methods have been developed to exploit neighbourhood spatial information in a local window, such as MR and NR. In (Zhuang et al., 2018), the authors proposed a spatialtemporal adaptive neighbourhood-based ratio to select optimal window size for solving the shortcoming of fixed-size rectangular window. Further, an adaptive generalized likelihood ratio test (AGLRT) was developed to weaken the geometric degradation of the DI caused by integrating heterogeneous pixels (Zhuang et al., 2020). Recently, Wang et al. (2020) developed a method of generating DI using irregular spatial information, in which the clear boundaries and textures were preserved by matching hypergraphs. 
One classical algorithm for DI analysis is $k$-means clustering. In (Celik., 2009), principal component analysis (PCA) feature extraction combined with $k$-means clustering was applied to assign each pixel of the 113 DI into the changed or unchanged class. Another typical algorithm is the FCM, which is more prevalent than 114 the former in SAR-image change detection (Gong et al., 2012; Gao et al., 2019; Li et al., 2019). The FCM clustered the Gabor feature vectors of the DI in SAR-based change detection (Li et al., 2015). To control the sensitivity to noise, a fuzzy local information c-means (FLICM) algorithm was developed by incorporating local spatial information and grey-level characteristics (Krinidis et al., 2010). Gong et al. (2012) employed it for change detection in SAR images. Tian et al. (2018) proposed an edge-weighted FCM by introducing a piecewise smooth prior to balance the trade-off between noise reduction and edge preservation. Li et al. (2019) developed a spatial FCM (SFCM) algorithm with a spatial function added into the fuzzy membership for noise suppression.

\subsection{Application of deep learning to change detection}

Recently, deep learning has been introduced to discriminate pixels of the intermediate class for SARbased change detection, thanks to its ability to learn high-level semantic and contextual features automatically. There are various deep neural networks available for change detection, such as PCANet, nonnegative- and Fisher-constrained autoencoder (NFCAE), CNN and CWNN (Gao et al., 2016; Geng et al., 2019; Li et al. 2019; Gao et al., 2019). CWNN is able to suppress speckle noise and suitable for processing SAR data by incorporating a wavelet-constrained pooling layer compared with a standard CNN (Duan et al., 2017). To train the deep learning model, a large amount of training samples is needed to ensure its classification performance. When training data are insufficient or imbalanced among all classes, data augmentation is usually adopted to handle the issue. Common augmentation techniques include affine transformations such as rotation, translation and scaling, as well as linear combination strategy (Shorten et al., 2019; Gao et al., 2019). However, these simple strategies are limited the generalisation capability of deep learning models (Frid-Adar et al., 2018). Generative adversarial networks (GANs) are powerful deep networks for training sample generation (Goodfellow et al., 2014; Shorten et al., 2019). The GAN model consists of two networks (generator and discriminator) that are trained in an adversarial fashion, where the 
generator creates fake images and the discriminator discriminates between real and fake images. Radford et

al. (2015) further developed a DCGAN, where both generator and discriminator were composed of deep

CNNs with fully convolution layers. The adversarial pair in DCGAN can learn a hierarchy of representations

both from image parts and entire images, making it superior to simple linear generation methods for data augmentation (Frid-Adar et al., 2018).

\section{The proposed approach}

Implementation of the proposed RUSACD approach is shown in Fig. 2. First, two SAR images are

filtered to denoise the image and enhance spatial features in a pre-processing stage, establishing a primary

DI through the LR operator. Superpixel-based reconstruction is performed on the primary DI in multi-scale

spaces, and the results fused into a MSRDI. Second, Gabor wavelet feature extraction is applied to the

MSRDI, with Gabor feature vectors acquired at the pixel level. Parallel TCCFCM clustering is conducted to

cluster these feature vectors as pseudo-labels (the changed, unchanged and intermediate classes). Third, the image patches together with pseudo-labels are obtained from two SAR images. The DCGAN is employed to enrich the pseudo-label image patches of the changed class. Finally, a CWNN is trained using these image patches with pseudo-labels, and is used to allocate the intermediate pixels to the changed or unchanged class. Thus, all pixels are marked with a unique label as changed or unchanged, obtaining the final change map. For clarity, pixels allocated to the changed and unchanged categories by the parallel TCCFCM are defined as simple pixels, since they can be discriminated easily. In contrast, intermediate pixels are treated as hard pixels due to the difficulty in discrimination by clustering alone.
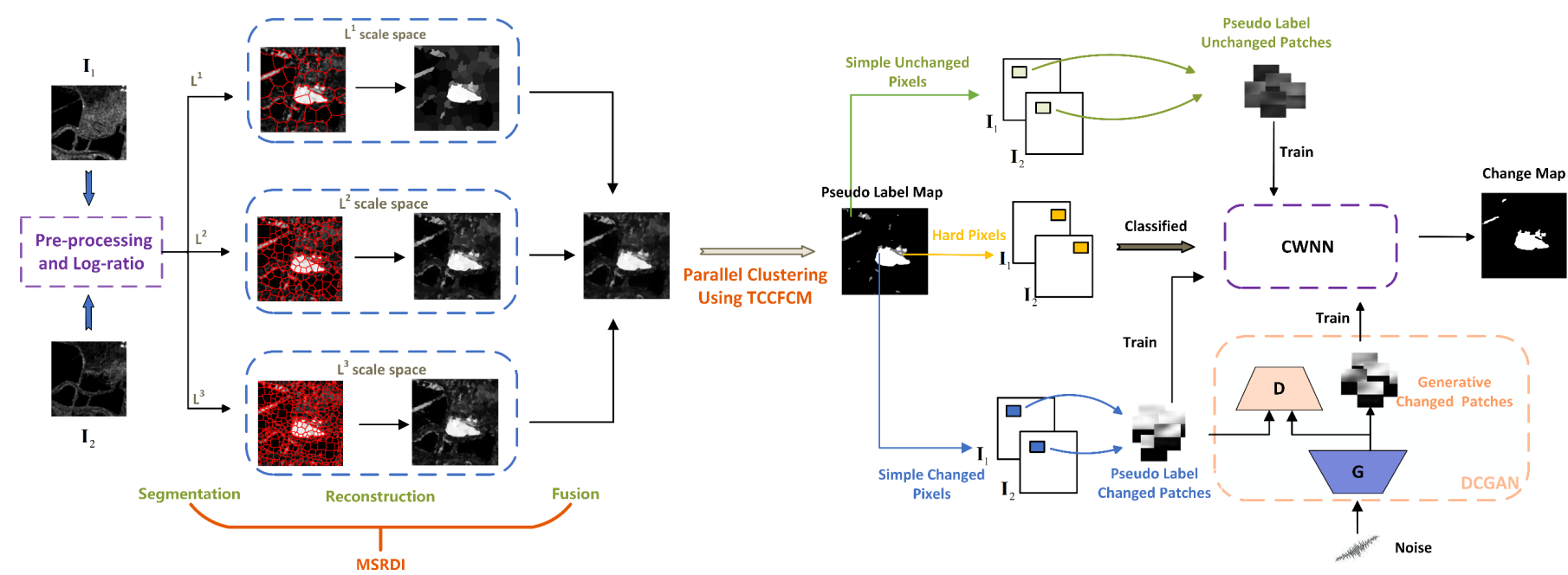

Fig. 2. Flowchart illustrating the proposed RUSACD methodology. Pseudo labels are drawn by three colours, black indicates unchanged pixels, white indicates changed pixels, and gray indicates intermediate (hard) pixels in Pseudo Label Map. 


\subsection{Pre-processing}

A weighted average filter is designed for pre-processing, which can suppress noise and enhance spatial domain features effectively. This filter $\mathbf{W}^{\eta}$ is defined as a $\eta \times \eta$ matrix, where $\eta$ is an odd number. Each element value of the matrix is determined by the distance between the current position and the centre of the matrix, as shown in Eq. (1). The centre of the matrix is defined as $w_{(\eta+1) / 2,(\eta+1) / 2}=2 / \eta^{2}$. The proposed filter maintains edges while suppressing speckle noise compared to other filters such as the mean filter, and retains the continuity of the image.

$$
w_{i j}=\frac{1}{\eta^{2} \sqrt{\left(\frac{\eta+1}{2}-i\right)^{2}+\left(\frac{\eta+1}{2}-j\right)^{2}}} \quad i, j=1,2, \ldots, \eta
$$

Given multi-temporal SAR images $\mathbf{I}_{1}$ and $\mathbf{I}_{2}$, where $\mathbf{I}_{1}, \mathbf{I}_{2} \in \mathbf{R}^{N_{r} \times N_{c}}, N_{r}$ and $N_{c}$ refer to the row and column of the images respectively. The convolution of each SAR image using $\mathbf{W}^{\eta}$ is taken to acquire two images $\mathbf{I}_{1}^{w}(\eta)=\mathbf{I}_{1} * \mathbf{W}^{\eta}$ and $\mathbf{I}_{2}^{w}(\eta)=\mathbf{I}_{2} * \mathbf{W}^{\eta}$, where $*$ denotes the 2-D convolution operation.

\subsection{Generating DI based on multi-scale superpixel reconstruction}

Simple Linear Iterative Clustering (SLIC) (Achanta et al., 2012) is used as the basis for superpixel segmentation. A multiscale superpixel reconstruction (MSRDI) is designed here for DI generation. The formal steps for MSRDI include:

Step 1: A log-ratio operator is used to generate the primary DI, and the log-ratio image $\mathbf{I}_{L R}$ is calculated as $\mathbf{I}_{L R}=\left|\log \left(\mathbf{I}_{2}^{w} / \mathbf{I}_{1}^{w}\right)\right|$.

Step 2: The filter template $\mathbf{W}^{\eta}$ is used to smooth the speckle in the log-ratio (LR) image $\mathbf{I}_{L R}$. The processed log-ratio image is described as $\mathbf{I}_{S L R}(\eta)=\mathbf{I}_{L R} * \mathbf{W}^{\eta}$.

Step 3: SLIC is used to split $\mathbf{I}_{S L R}$ into superpixels, each of which includes a number of pixels. Those superpixels are denoted as a set $\left\{\mathrm{S}_{l}\right\}_{l=1}^{l=L}$, where $l$ and $L$ represent the index and number of superpixels respectively. Each pixel in $\mathbf{I}_{S L R}$ is located in a corresponding superpixel $\mathrm{S}_{l}$. The median is an indicator to evaluate the level of a superpixel value, which is resistant to speckle noise. The mean contains homogeneous information of the corresponding superpixel. The mean value of each superpixel $o_{l}$ is obtained by calculating the average value of all pixels inside, which are collected into a set $\left\{o_{l}\right\}_{l=1}^{l=L}$. Similarly, the median of each superpixel $p_{l}$ can be calculated and collected into a set $\left\{p_{l}\right\}_{l=1}^{l=L}$. The superpixel-based DI is reconstructed pixel-by-pixel as 


$$
I_{S R D I}(i, j)=\alpha_{1} I_{i, j}+\alpha_{2} p_{l}+\alpha_{3} o_{l}
$$
details are well preserved.

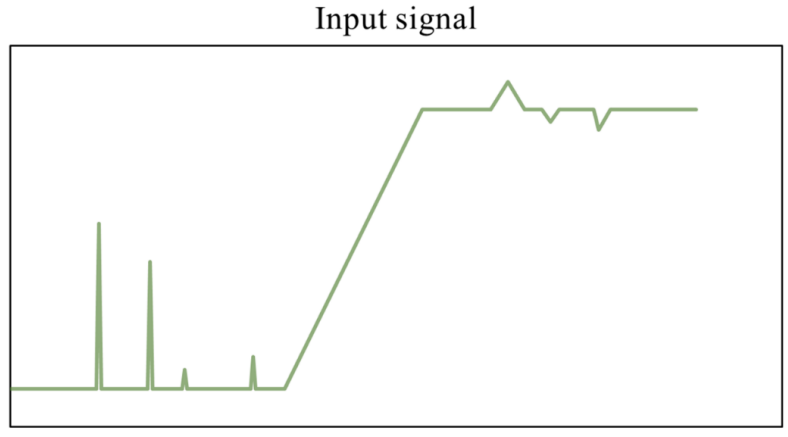

(a)

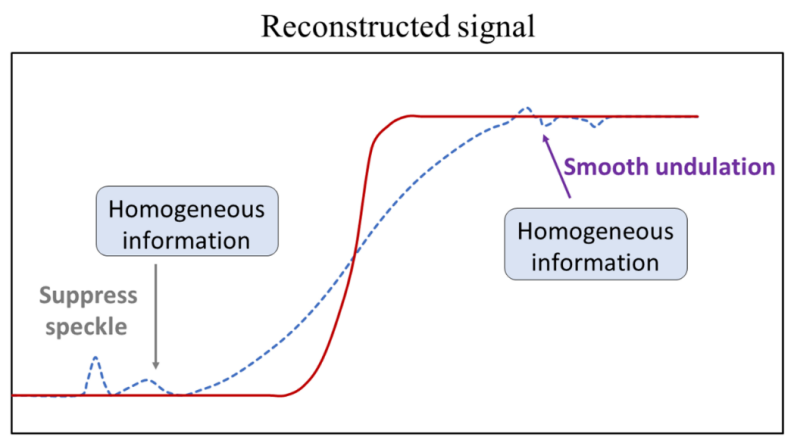

(c)

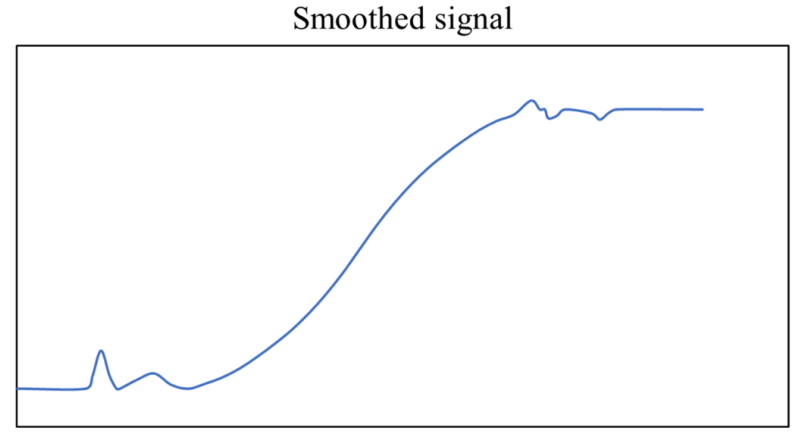

(b)

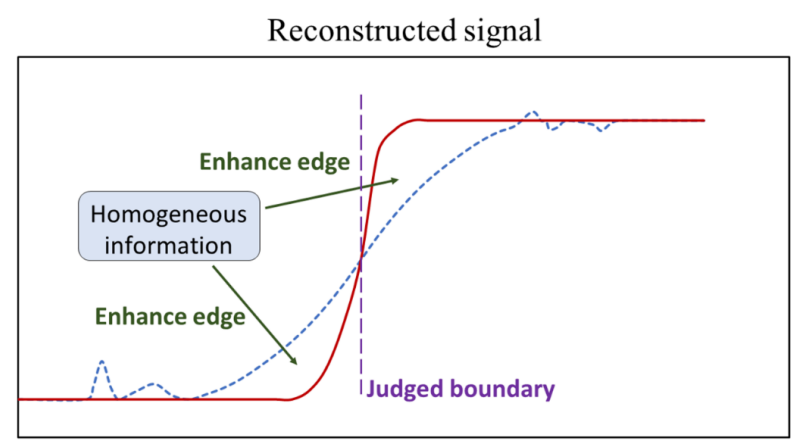

(d)

Fig. 3. Diagram using a one-dimensional signal to illustrate how MSRDI can minimise speckle noise and enhance edges.

(a) The input signal with speckle and distortion. (b) The smoothed signal using filter $\mathbf{W}^{\eta}$. (c) The reconstructed signal, in which noise is suppressed and distortion is mitigated. (d) The reconstructed signal, where the edges are enhanced. 


\subsection{Parallel TCCFCM clustering}

A two-stage centre-constrained FCM algorithm (TCCFCM) is designed for imbalanced data clustering to solve the small area change detection problem (Fig. 4). The first stage of TCCFCM is to find the preliminary and reliable clustering centres. The most important feature information provided by the DI is the pixel values: high-intensity pixels represent the changed class and low-intensity pixels refer to the unchanged class. Therefore, we select the top $N_{p}$ samples with prominent high-intensity $\left\{x_{p}^{h}\right\}_{p=1}^{N_{p}}$ and top $N_{p}$ samples with low $\left\{x_{p}^{l}\right\}_{p=1}^{N_{p}}$ values. These samples are then clustered by FCM to obtain cluster centres $v_{c}^{\text {pre }}$.

Those cluster centres are used as constraints in the second stage to prevent incorrect transfer of cluster prototypes (Fig. 4 (c) and (d)).

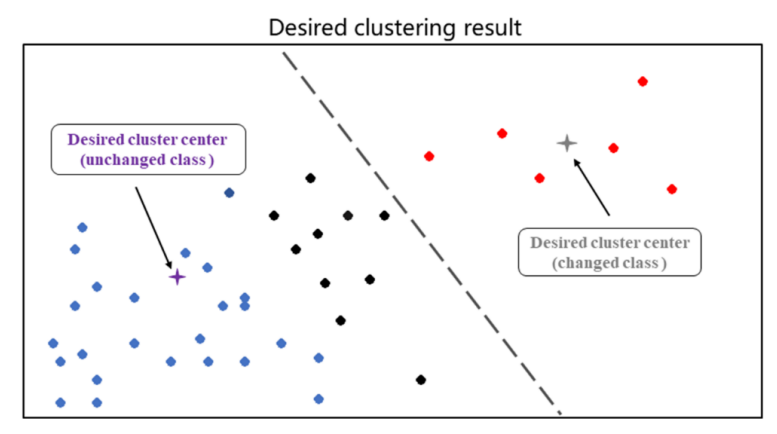

(a)

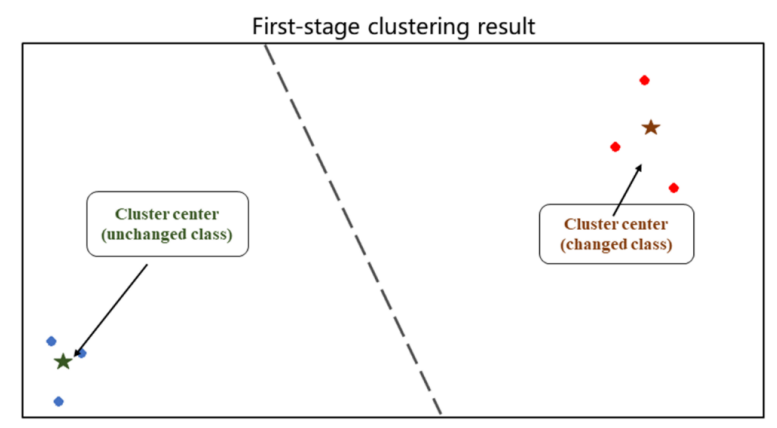

(c)

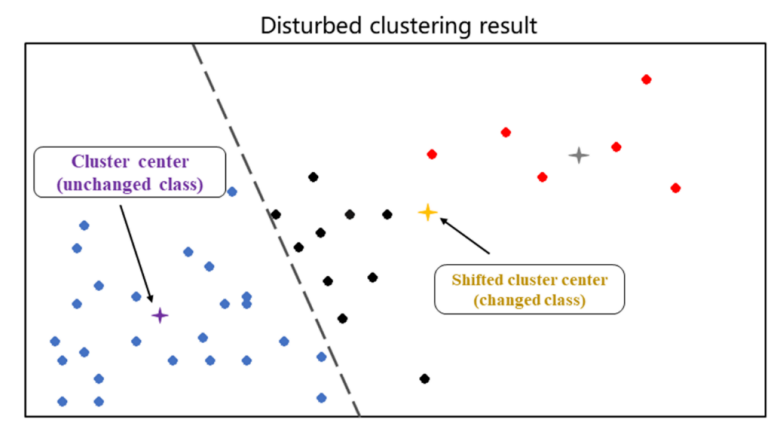

(b)

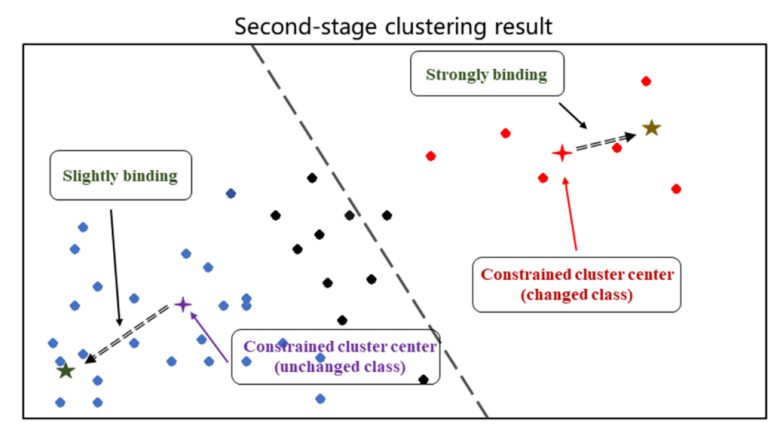

(d)

Fig. 4. Illustration of the principle of TCCFCM clustering. The red dots, blue dots and black dots represent pixels or samples of the changed class, low-intensity pixels or samples of the unchanged class, and medium-intensity pixels or samples of the unchanged class respectively. In (c), we obtain reliable clustering centres in the first stage. The reliable centres then constrain cluster prototypes to increase accuracy in the second stage, as shown in (d).

The second stage of TCCFCM performs clustering on all samples $\left\{x_{n}\right\}_{n=1}^{N_{r} N_{c}}$. The objective function is defined as:

$$
J=\sum_{c=1}^{2} \sum_{n=1}^{N_{r} N_{c}} u_{c n}^{m}\left\|\left(1-\beta_{c}\right) x_{n}+\beta_{c} v_{c}^{p r e}-v_{c}\right\|^{2}
$$

where $\beta_{c}$ is control parameter. We impose a strong constraint by setting a large value for the minority control parameter $\beta_{1}(c=1)$, and a small value for the majority control parameter $\beta_{2}(c=2)$ to impose a 
weak constraint. Hence, the two parameters are simplified as $\beta_{1}=\beta$ and $\beta_{2}=0.7 \cdot \beta$, which means that $\beta_{1}$ and $\beta_{2}$ are controlled uniformly by $\beta$. The element $u_{c n}$ of the membership partition matrix $\mathbf{U}$ and the cluster centre is derived as:

$$
\begin{gathered}
u_{c n}=\frac{1}{\sum_{j=1}^{2}\left(\frac{\left\|\left(1-\beta_{c}\right) x_{n}+\beta_{c} v_{c}^{p r e}-v_{c}\right\|^{2}}{\left\|\left(1-\beta_{j}\right) x_{n}+\beta_{j} v_{j}^{p r e}-v_{j}\right\|^{2}}\right)^{1 /(m-1)}} \\
v_{c}=\left(1-\beta_{c}\right) \frac{\sum_{n=1}^{N} u_{c n}^{m} x_{n}}{\sum_{n=1}^{N} u_{c n}^{m}}+\beta_{c} v_{c}^{p r e}
\end{gathered}
$$

where the initial membership partition matrix $\left(\mathbf{U}^{1}\right)$ is set randomly at the first stage, and the initial membership partition matrix $\left(\mathbf{U}^{2}\right)$ at the second stage is derived by the clustering centre $v_{c}^{\text {pre }}$.

A parallel clustering strategy based on TCCFCM is designed to discriminate simple pixels of both changed and unchanged classes, and hard pixels of the intermediate class. At the beginning of clustering, the sigmoid function is employed to perform two nonlinear mappings with different parameters on the MSRDI. One mapping prefers the significant changes, and the other prefers the weak changes. Then, a Gabor wavelet transform is used for feature extraction on the two mapped MSRDIs (Li et al., 2015; Gao et al, 2016), implemented by convolving $\mathbf{I}_{\mathrm{MSRDI}}$ with a set of Gabor kernels in eight directions on $\gamma$ scales. The maximum response is considered as a feature at this scale. The Gabor feature vector is expressed as $\mathbf{z}=\left[z_{0}, z_{1}, \ldots, z_{\gamma-1}\right]$. The details of the parallel TCCFCM clustering are as follows:

Step 1: Normalize and centralize the MSRDI $\mathbf{I}_{\mathrm{MSRDI}}$.

Step 2: Apply two sigmoid mappings to $\mathbf{I}_{\text {MSRDI }}$ with two different parameter sets at each pixel to achieve $\mathbf{I}_{1}^{M}=\operatorname{sigmoid}\left(\mathbf{I}_{\mathrm{MSRDI}} ; \mu_{1}\right)$ and $\mathbf{I}_{2}^{M}=\operatorname{sigmoid}\left(\mathbf{I}_{\mathrm{MSRDI}} ; \mu_{2}\right)$, where $\mu_{1}$ and $\mu_{2}$ represent two parameters. The sigmoid function is given by Eq. (7).

$$
\operatorname{sigmoid}(x ; \mu)=\frac{1}{1+e^{-(x+\mu)}}
$$

Step 3: Apply Gabor feature extraction to $\mathbf{I}_{1}^{M}$ and $\mathbf{I}_{2}^{M}$, and two Gabor feature vector sets are obtained, comprising $\mathbf{Z}^{1}=\left[\mathbf{z}_{1}^{1}, \mathbf{z}_{2}^{1}, \ldots, \mathbf{z}_{N_{r} N_{c}}^{1}\right]$ and $\mathbf{Z}^{2}=\left[\mathbf{z}_{1}^{2}, \mathbf{z}_{2}^{2}, \ldots, \mathbf{z}_{N_{r} N_{c}}^{2}\right]$, where $\mathbf{z}_{i}^{j} \in \mathbf{R}^{\gamma} \quad\left(i=1,2, \ldots, N_{r} N_{c} ; j=1,2\right)$ represents a Gabor feature vector.

Step 4: TCCFCM is utilised to perform two-class clustering on $\mathbf{Z}^{1}$ and $\mathbf{Z}^{2}$ to obtain two label sets $\mathbf{Y}^{1}=\left[y_{1}^{1}, y_{2}^{1}, \ldots, y_{i}^{1}, \ldots, y_{N_{r} N_{c}}^{1}\right]$ and $\mathbf{Y}^{2}=\left[y_{1}^{2}, y_{2}^{2}, \ldots, y_{i}^{2}, \ldots, y_{N_{r} N_{c}}^{2}\right]$, respectively, where $y_{i}^{j}$ represents a label 
corresponding to a Gabor feature vector. The value of $y_{i}^{j}$ is either 0 or 1 . The simple average operation is used to encode the two label sets, obtaining the final label set $\mathbf{Y}=\left[y_{1}, y_{2}, \ldots, y_{s} \ldots, y_{N_{r} N_{c}}\right]$, where $y_{i}=\left(y_{i}^{1}+y_{i}^{2}\right) / 2$.

Step 5: If $y_{i}=1$, the corresponding pixel is assigned to the changed class $\omega_{c}$. If $y_{i}=0$, the corresponding pixel is assigned to the unchanged class $\omega_{u c}$. The others represent hard pixels assigned to the hard class $\omega_{h}$.

\subsection{Deep learning CWNN and DCGAN}

To increase the accuracy of change detection for a small area, a deep CWNN is constructed to classify hard pixels. This is augmented by a DCGAN to increase the sample size for the rare changed class. A patch of size $\lambda \times 2 \lambda$ is selected, centred on a pixel of the changed class pseudo-label in the SAR image $\mathbf{I}_{1}$. Another patch is acquired in the same position from the SAR image $\mathbf{I}_{2}$ in the same way. Both patches are concatenated into a new patch with $2 \lambda \times 2 \lambda$ size as a pseudo-label training sample of the changed class. All pseudo-label training samples are produced according to the above method, denoted as $\left\{\mathbf{P}_{\tau_{1}}^{c}\right\}_{\tau_{1}=1}^{\tau_{1}=N_{1}}$ and $\left\{\mathbf{P}_{\tau_{2}}^{u c}\right\}_{\tau_{2}=1}^{\tau_{2}=N_{2}}$, where $\mathbf{P}_{\tau_{1}}^{c}, \mathbf{P}_{\tau_{2}}^{u c} \in \mathbf{R}^{2 \lambda \times 2 \lambda}$, representing the changed and unchanged patches, respectively. Hard patches $\left\{\mathbf{P}_{q}^{h}\right\}_{q=1}^{q=N_{h}}$ relative to hard pixels are obtained in the same way. Thereafter, a DCGAN is used to enrich the pseudo-label training samples of the changed class. The input vectors $\boldsymbol{\varphi}$ are initialized using random Gaussian noise, and are fed into the generator $G(\cdot)$ to generate false images, and the discriminator $D(\cdot)$ is built to distinguish the fake images from false and real images. Here, a convolutional generative model $G(\cdot)$ is built, with an objective max-min function as:

$$
\begin{aligned}
V(G, D) & =L^{f}+L^{r} \\
& =\mathbf{E}[\log (1-D(G(\boldsymbol{\varphi})))]+\mathbf{E}\left[\log \left(D\left(\mathbf{P}_{\tau_{1}}^{c}\right)\right)\right] \\
G^{*}= & \arg \min _{G} \max _{D} V(G, D)
\end{aligned}
$$

The structure of the designed deep learning model is illustrated in Fig. 5. The trained generator $G^{*}$ is used to expand the data to $\left\{\mathbf{P}_{\tau}^{c}\right\}_{\tau=1}^{\tau=N_{T}}$, in which $N_{T}-N_{1}$ image patches are generated as fake image patches. $2 N_{T}$ samples are selected to form a training dataset $\left\{\mathbf{P}_{\tau}^{c}, \mathbf{P}_{\tau}^{u c}\right\}_{\tau=1}^{\tau=N_{T}}$, where $\mathbf{P}_{\tau}^{c}, \mathbf{P}_{\tau}^{u c} \in \mathbf{R}^{2 \lambda \times 2 \lambda}$. The corresponding label $\left\{L_{\tau}^{c}, L_{\tau}^{u c}\right\}_{\tau=1}^{\tau=N_{T}}$ is defined by the parallel TCCFCM clustering result. All the pseudo-label training samples and labels are fed into the CWNN to classify the hard patches, where hard samples $\left\{\mathbf{P}_{q}^{h}\right\}_{q=1}^{q=N_{h}}$ are classified into changed and unchanged categories. The final change map can be obtained by combining the labels of hard pixels and simple pixels. 


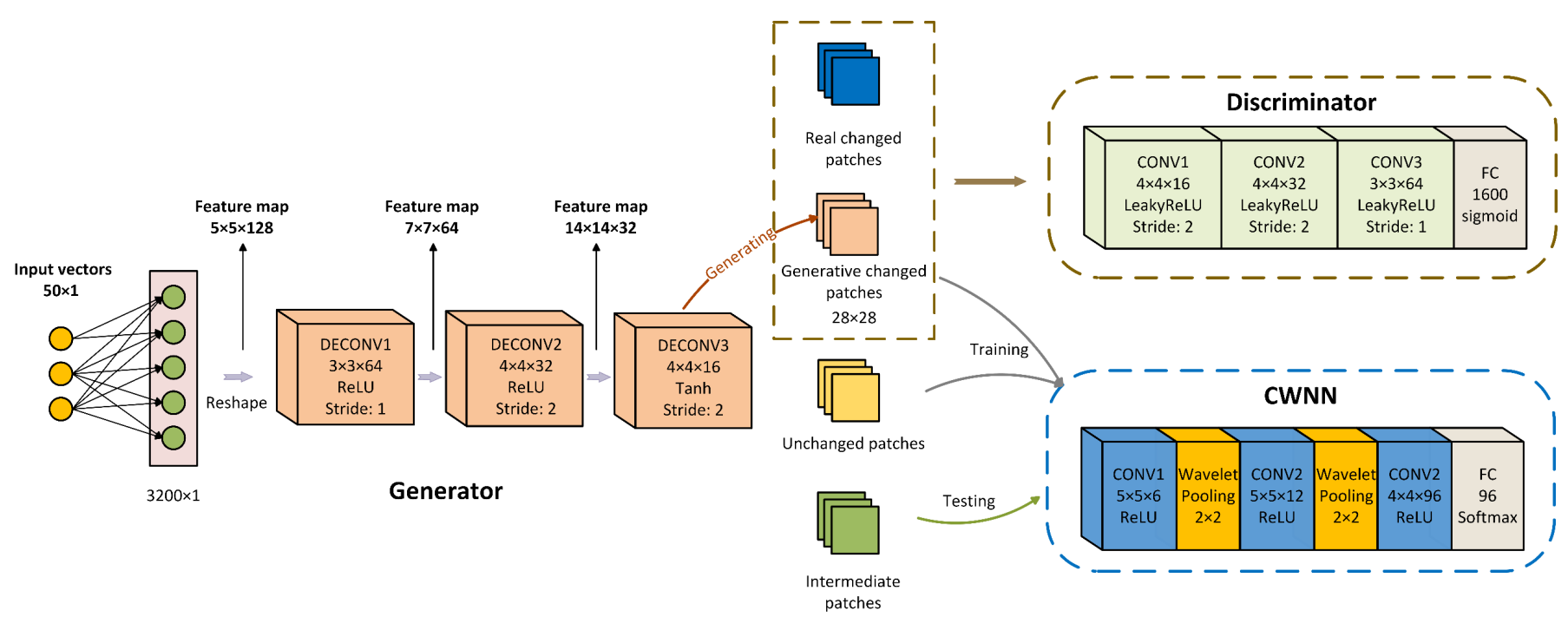

Fig. 5. Network structure of DCGAN and CWNN. Padding is employed in DECONV2, DECONV3, CONV1 and CONV2 in the generator and discriminator, where CONV and DECONV represent the convolutional layer and deconvolutional layer, respectively. FC is the fully connected layer.

\section{Numerical experiments}

\subsection{Experimental datasets}

Six real multi-temporal SAR datasets were used to evaluate the performance of the proposed approach. Three of these six datasets were acquired over the province of Guizhou in China by the COSMO-SkyMed SAR sensor on June 2016 and April 2017. As shown in Fig. 6, the first image pair (dataset A) with ground reference map consists of mountains and a river, the second image pair (dataset B) with ground reference map includes hills, plains and buildings, and the third image pair (dataset C) with ground reference map is mostly plains. Image pair of dataset D was acquired over the city of San Francisco, USA by the ERS-2 SAR sensor in August 2003 and May 2004 (Fig. 7). Image pair of dataset E (relating to Farmland C of Yellow River Estuary) was acquired by the Radarsat-2 satellite in June 2008 and June 2009 (Fig. 8), and Image pair of dataset F (relating to Ottawa flood) was acquired by Radarsat-1 satellite in May 1997 and August 1997 (Fig. 9). Speckle noise is shown in datasets A, B and C, which is very challenging for change detection. From the ground reference maps in Fig. 6, it is clear that the proportion of changed pixels is small compared with unchanged pixels. The detials are recorded in Table 1. In contrast, datasets D, E and F are relatively balanced with lower speckle noise than the former three datasets, which are used as benchmarks to evaluate the robustness and effectiveness of our approach. In this reserch, $N_{c}$ and $N_{u c}$ refer to the number of changed and unchanged pixels in the ground reference map, respectively. 
A

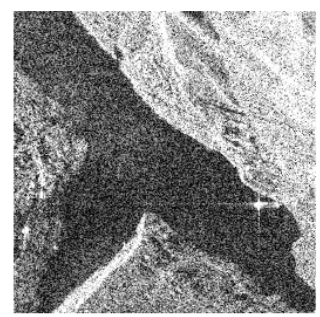

B

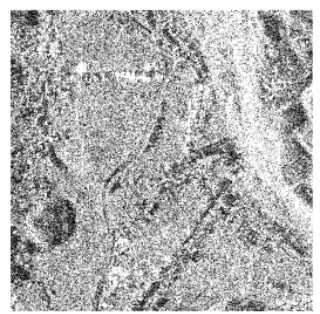

C

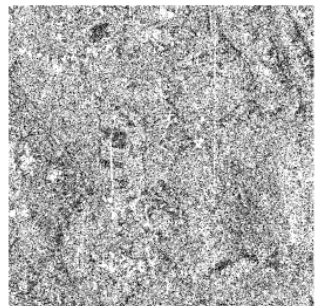

(a)
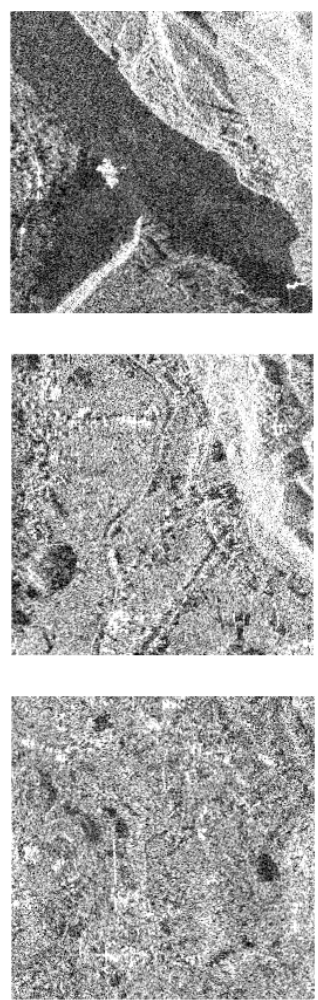

(b)
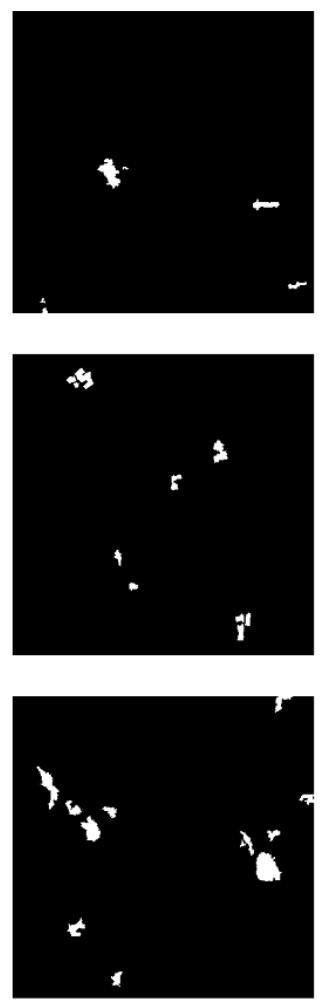

(c)

Fig. 6. Dataset A, B and C. (a) Image acquired in June 2016, (b) Image acquired in April 2017. (c) Ground reference map

D

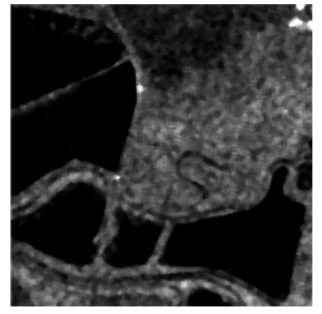

(a)

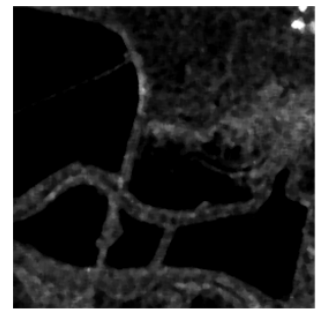

(b)

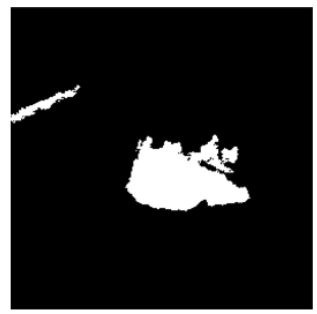

(c)

Fig. 7. Dataset D. (a) Image acquired in August 2003. (b) Image acquired in May 2004. (c) Ground reference map.

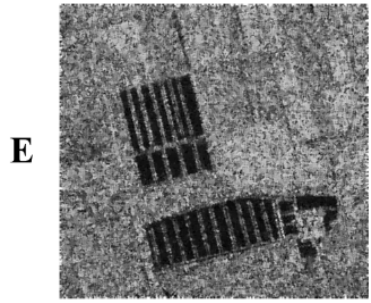

(a)

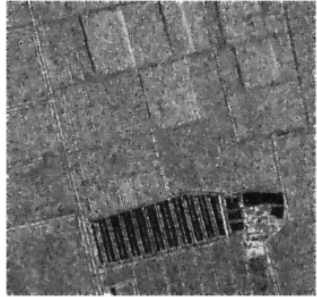

(b)

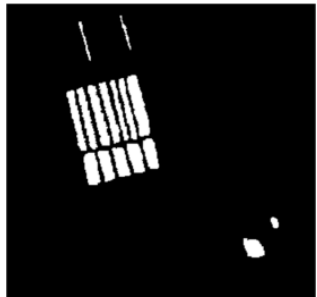

(c)

Fig. 8. Dataset E. (a) Image acquired in June 2008. (b) Image acquired in June 2009. (c) Ground reference map.

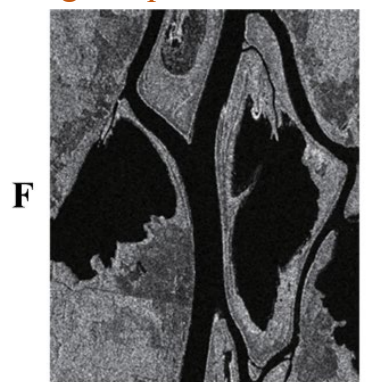

(a)

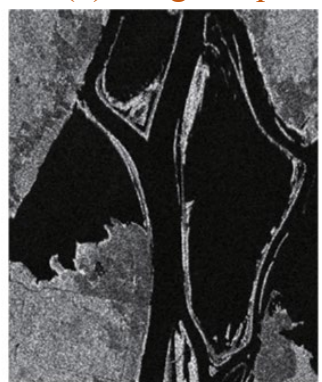

(b)

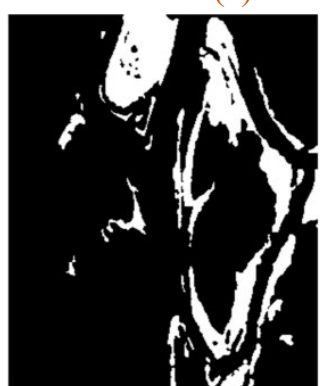

(c)

Fig. 9. Dataset F. (a) Image acquired in July 1997. (b) Image acquired in August 1997. (c) Ground reference map. 
Table 1. Summary of the experimental datasets.

\begin{tabular}{ccccc}
\hline Datasets & Size & $N_{c}$ & $N_{u c}$ & $N_{c}: N_{u c}$ \\
\hline A & $400 \times 400$ & 1066 & 158934 & $1: 149$ \\
B & $400 \times 400$ & 1492 & 158508 & $1: 106$ \\
C & $400 \times 400$ & 3467 & 156533 & $1: 45$ \\
D & $256 \times 256$ & 4685 & 60851 & $1: 13$ \\
E & $293 \times 308$ & 5378 & 84866 & $1: 16$ \\
F & $350 \times 290$ & 16049 & 85428 & $1: 5$ \\
\hline
\end{tabular}

306

\subsection{Evaluation criteria}

Five quantitative indicators were used to test the performance of the change detection methods: false alarm (FA) rate, missed detection (MD) rate, percentage correct classification (PCC), Kappa coefficient $(\mathrm{KC})$, and $\mathrm{F}_{1}$ score. The changed pixels are designated as positive and the unchanged pixels are assigned as negative, then, the confusion matrix is constructed. The false positive $(F P)$ denotes the number of unchanged pixels detected as the changed class, and the false negative $(F N)$ refers the number of changed pixels misclassified as unchanged. The true negative $(T N)$ and true positive $(T P)$ indicates the number of changed and unchanged pixel correctly detected respectively.

1) FA: The false alarm rate is given by $\mathrm{P}_{\mathrm{FA}}=\frac{F P}{F P+T P} \times 100 \%$.

2) $\mathrm{MD}$ : The missed detection rate is calculated as $\mathrm{P}_{\mathrm{MD}}=\frac{F N}{F N+T P} \times 100 \%$.

3) PCC: Accuracy of pixel-based classification can be expressed as

$$
\mathrm{PCC}=\frac{T P+T N}{T P+F P+T N+F N}
$$

4) KC: Kappa coefficient is used for consistency checks, defined as

$$
\begin{gathered}
\mathrm{KC}=\frac{\mathrm{PCC}-\mathrm{PRE}}{1-\mathrm{PRE}} \\
\mathrm{PRE}=\frac{(T P+F N) \times(T P+F P)+(T N+F P) \times(T N+F N)}{(T P+F N+T N+F P)^{2}}
\end{gathered}
$$

5) $\mathbf{F}_{1}: \mathrm{F}_{1}$ score is an essential indicator of classification performance, which is defined as

$$
\begin{aligned}
\mathrm{F}_{1} & =\frac{2 \times \text { precision } \times \text { recall }}{\text { precision }+ \text { recall }} \\
\text { precision } & =\frac{T P}{T P+F P} \quad \text { recall }=\frac{T P}{T P+F N}
\end{aligned}
$$




\subsection{Experimental setup and parameter setting}

All experiments were implemented on a PC with a 3.3-GHz four-core CPU and 24-GB memory. The GAN training and sample augmentation were implemented with NVIDIA GeForce RTX 2080s GPU with 8GB memory and PyTorch1.7.0. The CWNN training and testing were implemented using CPU and MATLAB2020a.

The hyper-parameters for each module were set separately. The weighted average filter kernel $\mathbf{W}^{\eta}$ size was set to $\eta=3$. In MSRDI, SLIC was conducted on four scales ( $\mathrm{T}=4$ ), and $L^{1}=100, L^{2}=500$, $L^{3}=1000, L^{4}=2000$ were used to obtain spatial information at the different scales on dataset A-D. $L^{1}=4000, L^{2}=8000, L^{3}=16000, L^{4}=32000$ were used for datasets E and F. Two sigmoid mapping parameters $\mu_{1}=-0.2$ and $\mu_{2}=0.3$ were set for unchanged and changed classes, respectively. $\mu_{2}$ was larger than 0.2, which ensured the reliable samples fed into the DCGAN. The Gabor feature extraction dimension was $\gamma=6$, and the constraint parameter of TCCFCM was set as $\beta=0.5$. Finally, the size of the image patch was chosen as $\lambda=14$.

The training of the DCGAN was as follows: 1) the Adam optimiser with a learning rate of 0.0003 was used to optimise the generator, and a learning rate of 0.0006 to optimise the discriminator. 2) Each iteration gave the real image a random value in the range 0.8 to 1 as a score, and the score of the fake image was provided randomly between 0 and 0.2 . The number of epochs was 10000 , and 640 image patches were selected and divided into 10 batches for training. A total of 4000 pseudo-label samples were used to train the CWNN with a learning rate of 0.0001 , and epochs set as 50 .

\subsection{Model complexity analysis}

In the MSRDI module, the complexity of the reconstruction method is linear in the number of pixels, with $O(N)$ complexity similar to SLIC. The proposed TCCFCM introduces cluster prototype constraints without increasing the computational complexity compared with standard FCM. The TCCFCM has $O\left(N I \rho c^{2}\right)$ complex, where $N$ represents the number of pixels, $I$ denotes the number of iterations and $\rho$ and $c$ refer to the dimensions of the pixel sample and number of clusters, respectively. The complexity of the deep learning models is listed in Table 2. In addition, 640 image patches of size $28 \times 28$ pixels were used for CWNN training, and employed for testing the forward propagation time. The same strategy was applied to record the running time of DCGAN. 
Table 2. Description of the deep learning models illustrating their complexity. K and M represent thousands and millions, respectively, FLOPs refer to floating point operations and MB is Megabytes.

\begin{tabular}{cccccc}
\hline Networks & Parameters (K) & Model size (MB) & FLOPs (M) & Forward time (s) & Training time (s / epoch) \\
\hline CWNN & 20.88 & 0.163 & 0.247 & 1.54 & 2.56 \\
Generator & 278 & 2.224 & 16.8 & 0.19 & 0.52 \\
Discriminator & 30.192 & 0.242 & 0.922 & 0.012 & \\
\hline
\end{tabular}

\section{Results and discussion}

\subsection{Results and comparison}

To demonstrate the effectiveness of the proposed RUSACD, four benchmark methods were compared, including: PCA k-means (PCAK) (Celik, 2009), neighbourhood ratio and extreme learning machine (NRELM) (Gao et al., 2016), Gabor feature extraction and FCM with PCANet (GFPCANet) (Gao et al., 2016), and FCM with CWNN (FCWNN) (Gao et al., 2019). We also applied TCCFCM to cluster the MSRDI into two categories (changed and unchanged) as a simple, fast and effective variant change detection method MTCCFCM. The experimental results are shown in Fig. 8, while the quantitative accuracy metrics are given in Table 3. In addition, other methods used for comparison on datasets D, E and F (Table 4, 5, 6) include saliency-guided detection with $k$-means clustering (SGK) (Zheng et al., 2017), stacked autoencoder and FCM with CNN (SAEFCNN) (Gong et al., 2017), saliency-guided deep neural network (SGDNN) (Geng et al., 2019), adaptive generalised likelihood ratio test (AGLRT) (Zhuang et al., 2020), PCANet with Saliency detection (SDPCANet) (Li et al., 2019), spatial FCM and CNN (SFCNN) (Li et al., 2019), FCM and Deep Belief Network (FDBN) (Gong et al., 2016) and hypergraph-based change detection framework (HCDF) (Wang et al., 2020), the numerical results of these methods are acquired from published articles.

From Table 3, RUSACD could maintain a change detection accuracy of more than $99 \%$, which is the best among all the methods. The $\mathrm{F}_{1}$ and $\mathrm{KC}$ of RUSACD are greater than $70 \%$, which is significantly superior to the other methods. Both PCAK and NRELM produced a low accuracy due to the high sensitivity of kmeans clustering and extreme learning machine (ELM) to noise. Datasets A, B and C contained massive speckle noise, which led to a very large number of false alarms with limited accuracy. The change detection accuracy for GFPCANet and FCWNN is low since the lack of guiding information in DI and clustering labels. For dataset D (with low noise), most approaches showed excellent detection capabilities, except for PCAK. For the proposed RUSACD, the PCC reached the highest accuracy of $99.24 \%$, and both $\mathrm{F}_{1}$ and KC exceeded 94\%. The MTCCFCM achieved competitive results compared with methods based on deep 
376 learning. It can be concluded that RUSACD is very effective for small area change detection from multi377 temporal SAR images with strong speckle noise.

In addition, RUSACD also exhibits outstanding detection performance on benchmark datasets as shown

in Table 5 and Table 6. For dataset E, RUSACD achieves best performance on PCC, $\mathrm{F}_{1}$ and $\mathrm{KC}$ among all methods. Although the performance of RUSACD is not best on dataset F, it achieves competitive performance compared with other state-of-the-art methods. The MSRDI is designed for SAR images with strong speckle noise, and it restrains some tiny discrete changed pixels as noise on dataset F.
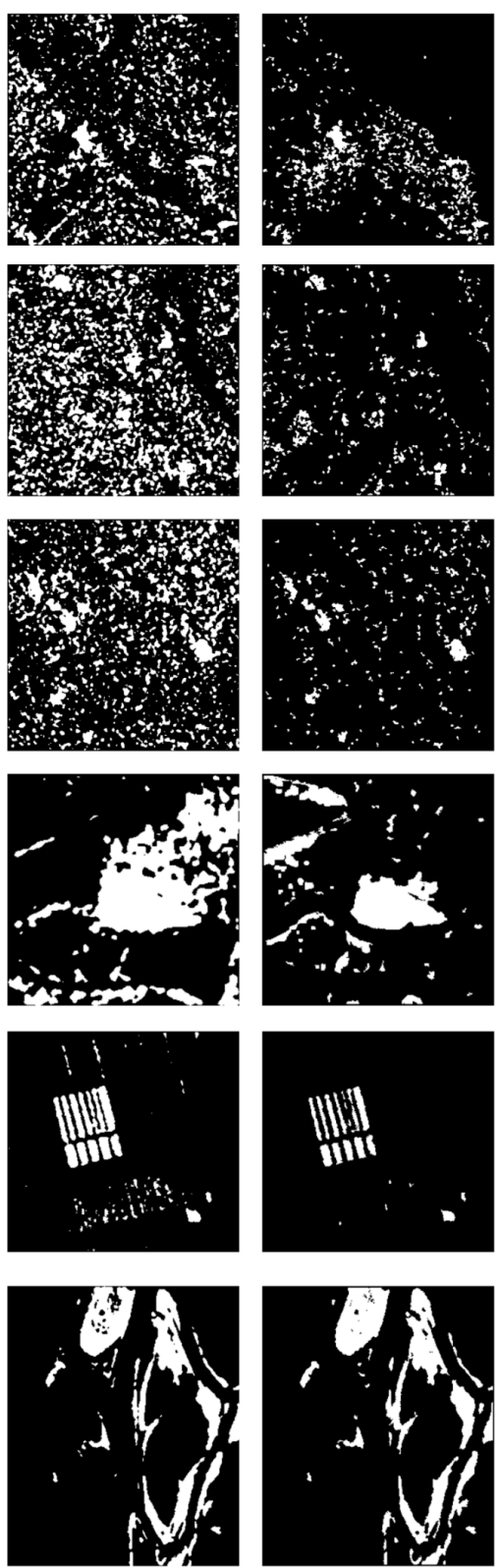

(a)
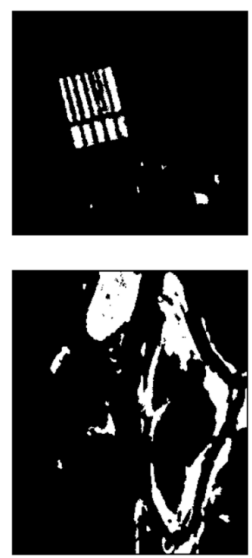

(b)
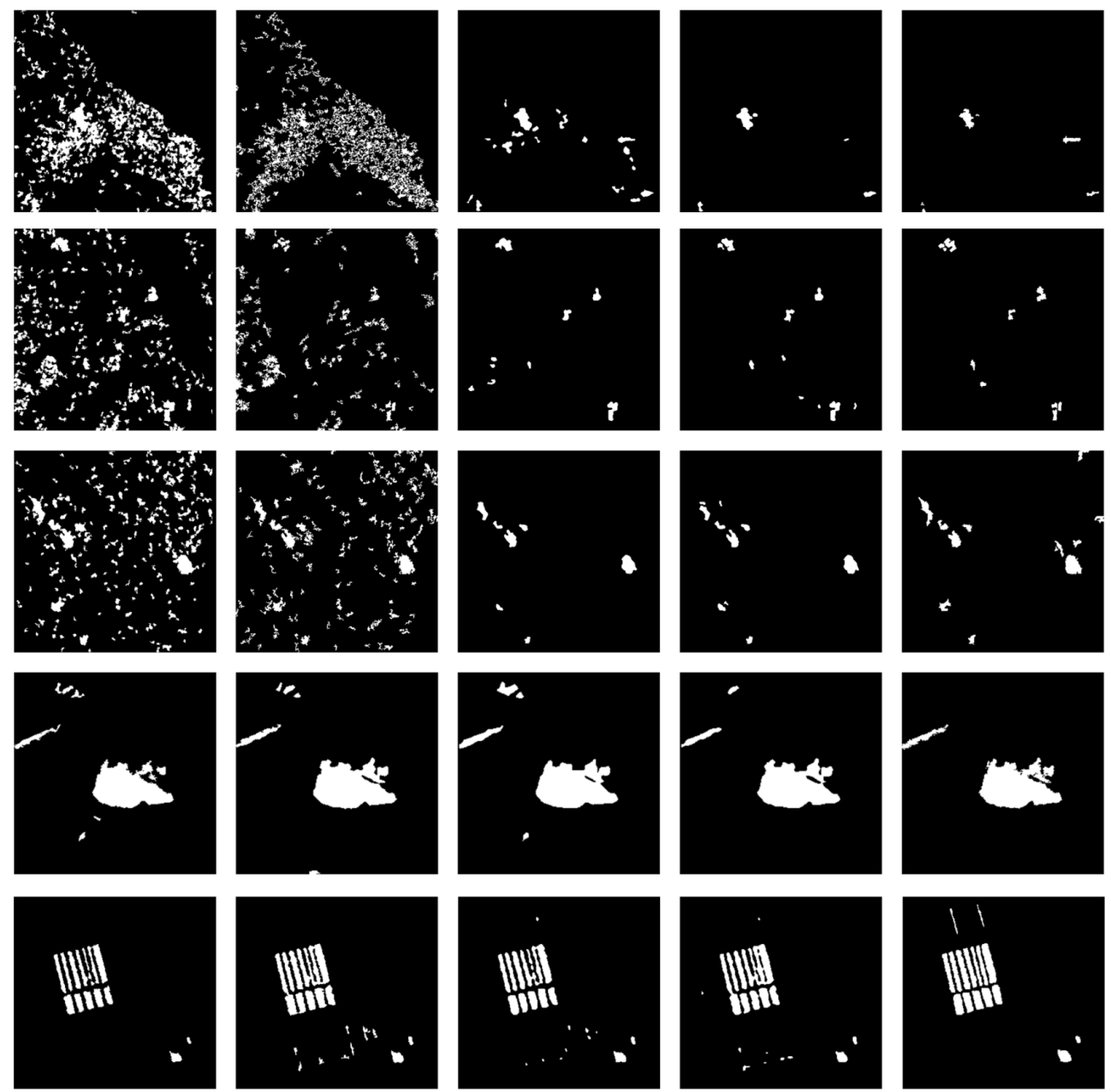

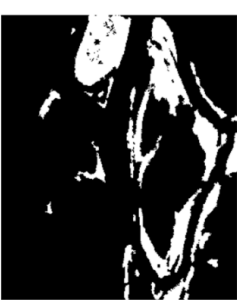

(c)

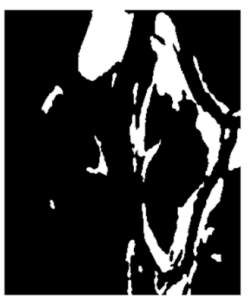

(d)

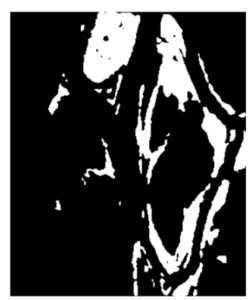

(e)

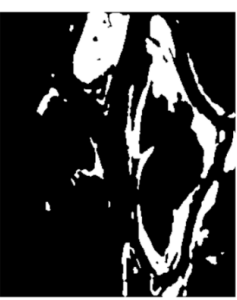

(f)
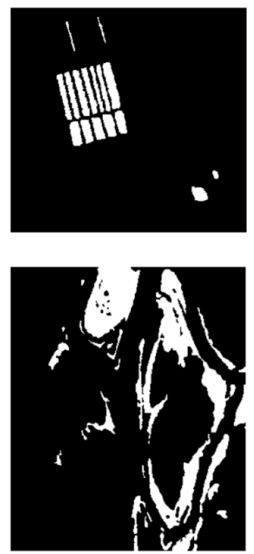

(g)

Fig. 10. Comparison of the final change maps on all datasets. (a) PCAK. (b) NRELM. (c) GFPCANet. (d) FCWNN. (e) MTCCFCM. (f) RUSACD. (g) Ground reference map. 
Table 3. Accuracy metrics for the different change detection methods. Best results are shown in bold.

\begin{tabular}{|c|c|c|c|c|c|c|c|c|}
\hline \multicolumn{2}{|c|}{ Criterion } & FP & FN & $\mathrm{P}_{\mathrm{FA}}(\%)$ & $\mathrm{P}_{\mathrm{MD}}(\%)$ & PCC (\%) & $\mathrm{KC}(\%)$ & $F_{1}(\%)$ \\
\hline \multirow{6}{*}{ Dataset A } & PCAK & 27822 & 1 & 96.45 & 0.1 & 82.61 & 5.69 & 6.86 \\
\hline & NRELM & 11312 & 42 & 92.00 & 4.10 & 92.90 & 13.74 & 14.76 \\
\hline & GFPCANet & 20935 & 9 & 95.37 & 0.88 & 86.91 & 7.71 & 8.84 \\
\hline & FCWNN & 18108 & 383 & 96.58 & 37.37 & 88.44 & 5.34 & 6.49 \\
\hline & MTCCFCM & 2118 & 107 & 69.76 & 10.44 & 98.61 & 44.68 & 45.21 \\
\hline & RUSACD & 390 & 241 & 33.22 & 23.51 & 99.61 & 71.11 & 71.31 \\
\hline \multirow{6}{*}{ Dataset B } & PCAK & 43095 & 47 & 96.76 & 3.15 & 73.04 & 4.56 & 6.28 \\
\hline & NRELM & 8066 & 208 & 86.27 & 13.94 & 94.83 & 22.44 & 23.69 \\
\hline & GFPCANet & 14553 & 102 & 91.28 & 6.84 & 90.84 & 14.49 & 15.94 \\
\hline & FCWNN & 7822 & 602 & 73.19 & 17.36 & 94.74 & 38.47 & 40.48 \\
\hline & MTCCFCM & 677 & 330 & 36.81 & 22.12 & 99.37 & 69.45 & 69.77 \\
\hline & RUSACD & 596 & 315 & 33.62 & 21.11 & 99.43 & 71.81 & 72.10 \\
\hline \multirow{6}{*}{ Dataset $\mathrm{C}$} & PCAK & 31048 & 241 & 90.59 & 6.95 & 80.44 & 13.70 & 17.10 \\
\hline & NRELM & 6527 & 729 & 70.45 & 21.03 & 95.47 & 41.15 & 43.01 \\
\hline & GFPCANet & 11583 & 331 & 78.69 & 9.55 & 92.55 & 32.11 & 34.49 \\
\hline & FCWNN & 7194 & 417 & 87.00 & 27.95 & 95.24 & 20.77 & 22.03 \\
\hline & MTCCFCM & 159 & 1459 & 7.37 & 42.08 & 98.99 & 70.79 & 71.28 \\
\hline & RUSACD & 196 & 1406 & 8.68 & 40.55 & 99.00 & 71.53 & 72.01 \\
\hline
\end{tabular}

Table 4. Accuracy metrics for the different change detection methods on dataset D. Best results are shown in bold.

\begin{tabular}{|c|c|c|c|c|c|c|c|}
\hline Criterion & FP & FN & $\mathrm{P}_{\mathrm{FA}}(\%)$ & $\mathrm{P}_{\mathrm{MD}}(\%)$ & PCC (\%) & $\mathrm{KC}(\%)$ & $F_{1}(\%)$ \\
\hline AGLRT & 231 & 552 & 5.27 & 11.78 & 98.80 & 91.10 & 91.80 \\
\hline SGK & 561 & 489 & 11.79 & 10.44 & 98.40 & 88.02 & 88.88 \\
\hline SAEFCNN & 231 & 604 & 5.36 & 12.89 & 98.73 & 90.04 & 90.72 \\
\hline SGDNN & 321 & 509 & 7.14 & 10.86 & 98.73 & 90.28 & 90.96 \\
\hline PCAK & 13784 & 182 & 75.38 & 3.88 & 78.69 & 31.40 & 39.20 \\
\hline NRELM & 4466 & 51 & 49.08 & 1.09 & 93.11 & 63.81 & 67.23 \\
\hline GFPCANet & 321 & 337 & 6.88 & 7.19 & 99.00 & 92.42 & 92.97 \\
\hline FCWNN & 545 & 150 & 10.73 & 3.20 & 98.94 & 92.31 & 92.88 \\
\hline MTCCFCM & 621 & 148 & 12.04 & 3.16 & 98.83 & 91.55 & 92.19 \\
\hline RUSACD & 279 & 217 & 5.88 & 4.63 & 99.24 & 94.33 & 94.74 \\
\hline
\end{tabular}

Table 5. Accuracy metrics for the different change detection methods on dataset E. Best results are shown in bold.

\begin{tabular}{cccccccc}
\hline Criterion & FP & FN & $\mathrm{P}_{\mathrm{FA}}(\%)$ & $\mathrm{P}_{\mathrm{MD}}(\%)$ & $\mathrm{PCC}(\%)$ & $\mathrm{KC}(\%)$ & $\mathrm{F}_{1}(\%)$ \\
\hline FDBN & 659 & $\mathbf{6 4 1}$ & 12.21 & $\mathbf{1 1 . 9 2}$ & 98.56 & 87.17 & 87.93 \\
SFCNN & 380 & 903 & 7.83 & 16.79 & 98.58 & 86.71 & 87.46 \\
PCAK & 1269 & 652 & 21.17 & 12.12 & 97.87 & 81.98 & 83.11 \\
NRELM & 76 & 1958 & 2.17 & 36.41 & 97.75 & 75.95 & 77.08 \\
GFPCANet & $\mathbf{2 4}$ & 1535 & $\mathbf{0 . 6 2}$ & 28.54 & 98.27 & 82.25 & 83.14 \\
FCWNN & 589 & 705 & 11.19 & 13.11 & 98.57 & 87.08 & 87.84 \\
MTCCFCM & 250 & 1013 & 5.42 & 18.84 & 98.60 & 86.62 & 87.36 \\
RUSACD & 360 & 837 & 7.35 & 15.56 & $\mathbf{9 8 . 6 7}$ & $\mathbf{8 7 . 6 5}$ & $\mathbf{8 8 . 3 5}$ \\
\hline
\end{tabular}


Table 6. Accuracy metrics for the different change detection methods on dataset F. Best results are shown in bold.

\begin{tabular}{cccccccc}
\hline Criterion & FP & FN & PFA $(\%)$ & PMD $(\%)$ & PCC $(\%)$ & KC $(\%)$ & F $(\%)$ \\
\hline AGLRT & 882 & 678 & 5.74 & 4.22 & 98.46 & 94.13 & 94.89 \\
SDPCANet & 820 & 944 & 5.15 & 5.88 & 98.26 & 93.45 & 94.48 \\
FDBN & $\mathbf{5 6 9}$ & 620 & $\mathbf{3 . 5 6}$ & 3.86 & 98.83 & 95.59 & 96.29 \\
SFCNN & $\mathbf{5 6 9}$ & 782 & 3.59 & 4.87 & 98.67 & 94.97 & 95.76 \\
HCDF & 615 & $\mathbf{5 1 6}$ & 3.81 & $\mathbf{3 . 2 2}$ & $\mathbf{9 8 . 8 9}$ & $\mathbf{9 5 . 8 3}$ & $\mathbf{9 6 . 4 9}$ \\
PCAK & 755 & 1718 & 5.00 & 10.70 & 97.56 & 90.62 & 92.06 \\
NRELM & 577 & 1690 & 3.86 & 10.53 & 97.77 & 91.37 & 92.68 \\
GFPCANet & 1047 & 821 & 6.43 & 5.12 & 98.16 & 93.13 & 94.22 \\
FCWNN & 956 & 933 & 5.95 & 5.81 & 98.14 & 93.01 & 94.12 \\
MTCCFCM & 1527 & 539 & 8.96 & 3.36 & 97.96 & 92.54 & 93.76 \\
RUSACD & 1335 & 560 & 7.94 & 3.49 & 98.13 & 93.12 \\
\hline
\end{tabular}

\subsection{Performance analysis of MSRDI}

Five DI generation methods were compared in the experiment: LR, SLR (smoothed LR by the proposed weighted average filter), NR, CDI (Zheng et al., 2014) and MSRDI. The results for Datasets C and D are shown in Fig. 11. Compared with the other DIs, the images in MSRDI are smooth, the edges are strengthened and the outliers are suppressed since MSRDI exploits the local spatial information in superpixels at multiple scales. MSRDI has a strong ability to suppress noise and retain clear edges, particularly for small area change detection (the first row of Fig. 11).
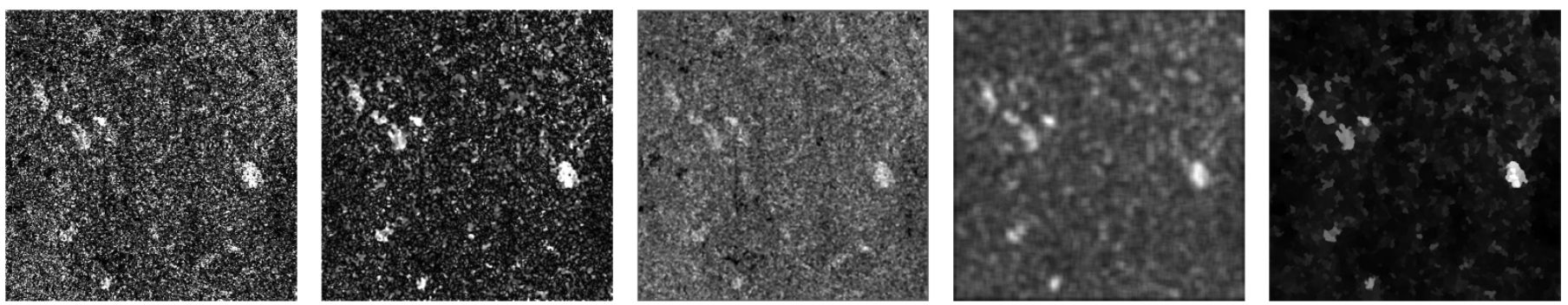

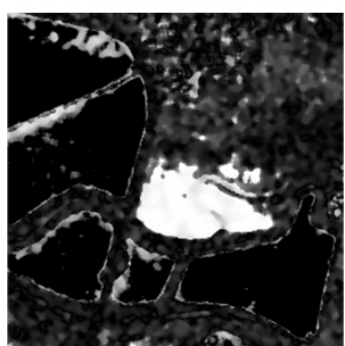

(a)

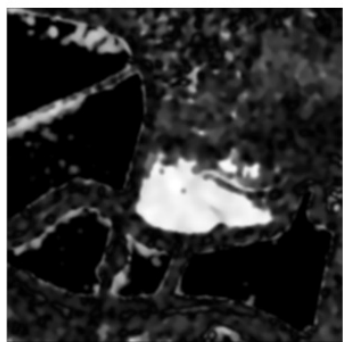

(b)

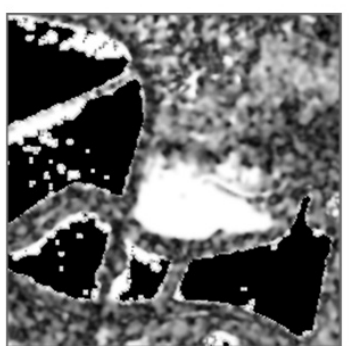

(c)

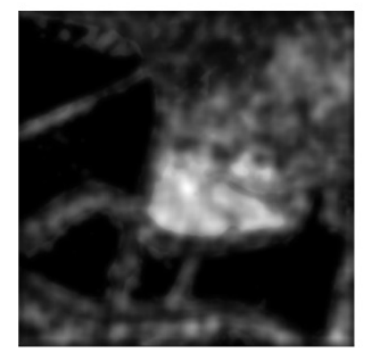

(d)

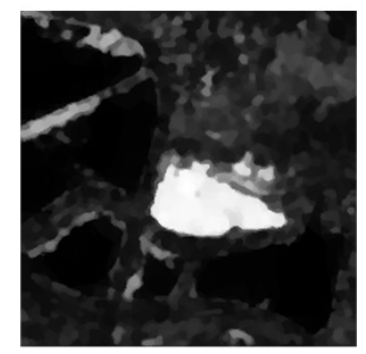

(e)

Fig. 11. DIs of the five methods on datasets C and D. (a) LR. (b) SLR. (c) NR. (d) CDI. (e) MSRDI.

The performance of MSRDI was further tested using the Otsu thresholding method to segment all DIs, as shown in Fig. 12 and Table 7. MSRDI is superior to the other methods, with the lowest false alarm rate and the highest accuracy. The NR-based DI and detection results are unsatisfactory for both datasets. For 
dataset $\mathrm{C}$ with strong speckle noise, the mean and median operators in MSRDI were beneficial for suppressing speckle noise. For Dataset D, MSRDI was able to enhance the edges and strengthen the separability of the DI, while smoothing the image.
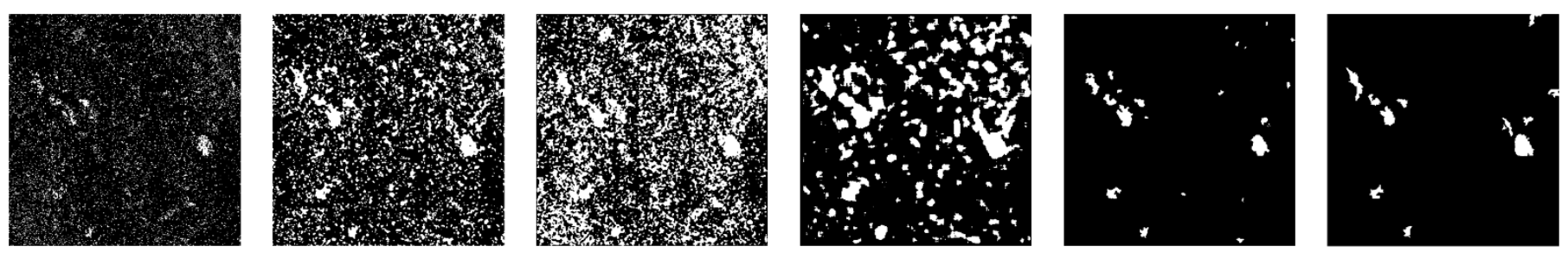

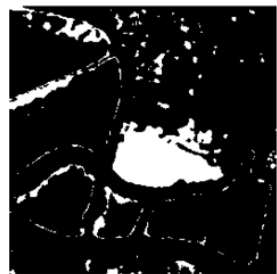

(a)

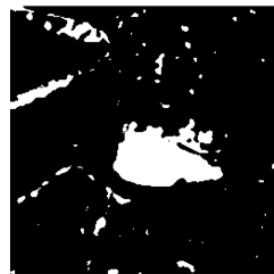

(b)

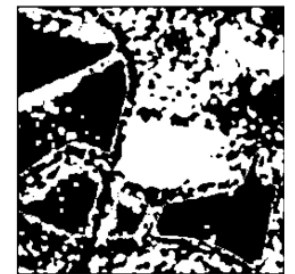

(c)

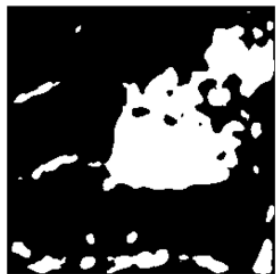

(d)

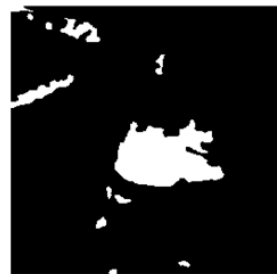

(e)

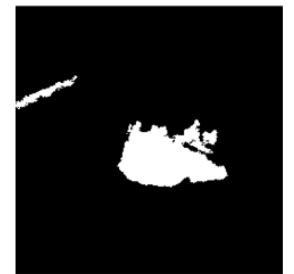

(f)

Fig. 12. Change maps of the five DI methods using Otsu thresholding on dataset $C$ and D. (a) LR. (b) SLR. (c) NR. (d) CDI. (e) MSRDI. (f) Ground reference map.

Table 7. Change detection accuracy metrics for five DIs acquired by Otsu thresholding. Best results are shown in bold.

\begin{tabular}{cccccc|ccccc}
\hline \multicolumn{3}{c}{ Dataset C } & \multicolumn{5}{c}{ Dataset D } \\
\hline Criterion & FP & FN & PCC (\%) & KC (\%) & F $_{1}(\%)$ & FP & FN & PCC (\%) & KC (\%) & F $(\%)$ \\
LR & 12083 & 2000 & 91.20 & 14.28 & 17.24 & 2917 & 146 & 95.33 & 72.34 & 74.77 \\
SLR & 30911 & 309 & 80.49 & 13.42 & 16.83 & 1952 & 130 & 96.82 & 79.71 & 81.40 \\
NR & 54044 & 236 & 66.08 & 6.83 & 10.64 & 23295 & $\mathbf{4}$ & 64.45 & 18.71 & 28.66 \\
CDI & 24977 & $\mathbf{1 5 3}$ & 84.29 & 17.69 & 20.87 & 11947 & 152 & 81.54 & 35.67 & 42.83 \\
MSRDI & $\mathbf{7 8 5}$ & 1110 & $\mathbf{9 8 . 8 2}$ & $\mathbf{7 0 . 7 2}$ & $\mathbf{7 1 . 3 3}$ & $\mathbf{1 2 3 7}$ & 174 & $\mathbf{9 7 . 8 5}$ & $\mathbf{8 5 . 3 2}$ & $\mathbf{8 6 . 4 8}$ \\
\hline
\end{tabular}

\subsection{Performance analysis of TCCFCM}

Six segmentation methods were used to segment the MSRDI of dataset B to achieve change maps, as shown in Fig. 13 and Table 8. Here, GTCCFCM represents segmentation based on Gabor feature extraction together with TCCFCM. GKM represents the combination of Gabor feature extraction and the k-means clustering algorithm. GFCM refers to Gabor feature extraction with FCM clustering. In addition, two methods were used as benchmarks, including classic FCM and TCCFCM algorithms with Gabor wavelet features extracted from LR images.

The change map of GTCCFCM produced reliable detection results, with the highest accuracy of 99.37\%, demonstrating robustness for small area change detection. Other methods such as FCM produced a significant number of false alarms. The k-means algorithm shows some benefits by minimising the incorrect transfer of cluster centres, although it still contains several medium-value unchanged pixels within the cluster 
prototype of changed class, leading to increased false alarms. Fig. 13(b) and the results of LGTCCFCM

demonstrate the effectiveness of the proposed algorithm, without overly relying on the high-quality MSRDI.

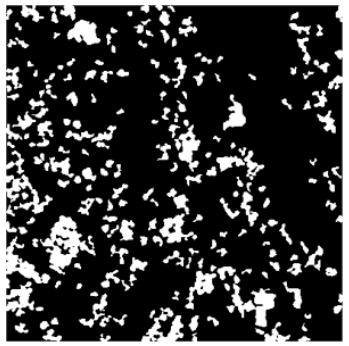

(a)

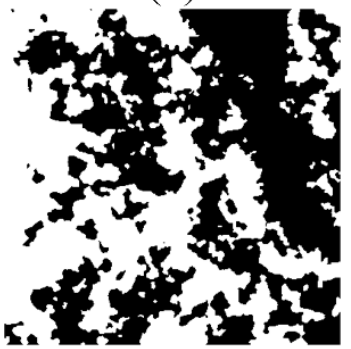

(f)

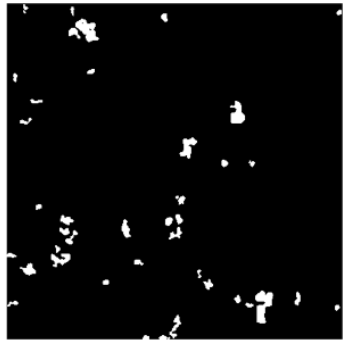

(b)

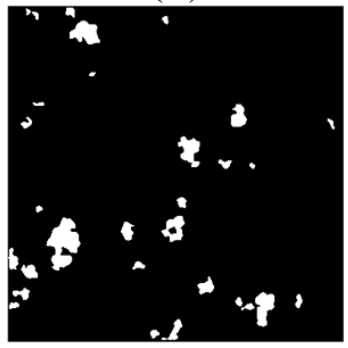

(g)

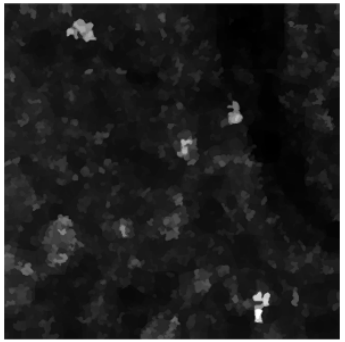

(c)

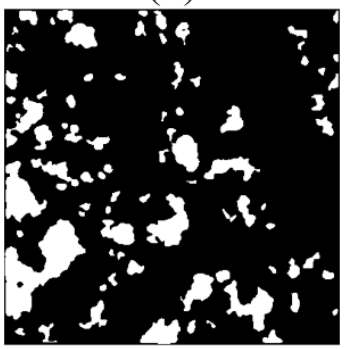

(h)

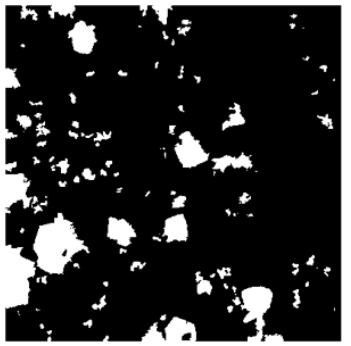

(d)

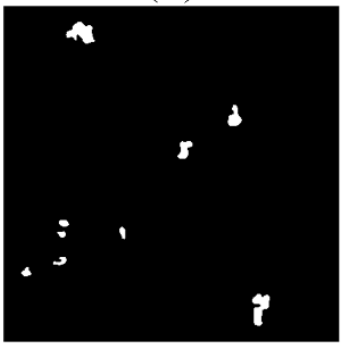

(i)

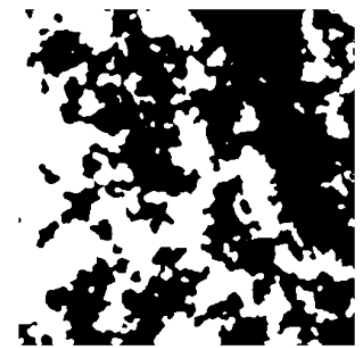

(e)

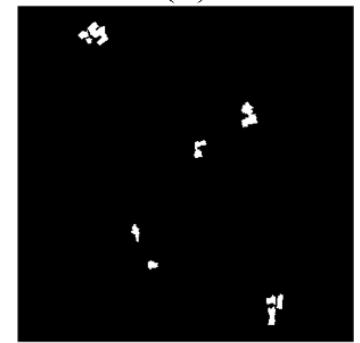

(j)

Fig. 13. Change maps of the image segmentation methods on dataset B. (a) LGFCM. (b) LGTCCFCM. (c) MSRDI. (d) Otsu threshold. (e) FLICM. (f) RFLICM. (g) GKM. (h) GFCM. (i) GTCCFCM. (j) Ground reference map.

Table 8. Change detection accuracy metrics acquired on dataset B. Best results are shown in bold.

\begin{tabular}{|c|c|c|c|c|c|c|c|}
\hline Criterion & FP & $\mathrm{FN}$ & $\mathrm{P}_{\mathrm{FA}}(\%)$ & $\mathrm{P}_{\mathrm{MD}}(\%)$ & PCC (\%) & $\mathrm{KC}(\%)$ & $\mathrm{F}_{1}(\%)$ \\
\hline LGFCM & 26718 & 44 & 94.86 & 2.95 & 83.27 & 8.14 & 9.76 \\
\hline LGTCCFCM & 2614 & 222 & 67.30 & 14.88 & 98.23 & 46.53 & 47.25 \\
\hline Otsu & 17522 & 126 & 92.77 & 8.45 & 88.97 & 11.88 & 13.41 \\
\hline FLICM & 70990 & 3 & 97.95 & 0.2 & 55.63 & 2.24 & 4.03 \\
\hline RFLICM & 78150 & 25 & 98.16 & 1.68 & 51.14 & 1.82 & 3.62 \\
\hline GKM & 5272 & 128 & 79.45 & 8.58 & 96.63 & 32.54 & 33.56 \\
\hline GFCM & 24242 & 32 & 94.32 & 2.14 & 84.83 & 9.14 & 10.74 \\
\hline GTCCFCM & 677 & 330 & 36.81 & 22.12 & 99.37 & 69.45 & 69.77 \\
\hline
\end{tabular}

Table 9. Simple pixel classification accuracy by the proposed parallel TCCFCM clustering strategy. The $\mathrm{PCC}_{\mathrm{c}}$ and $\mathrm{PCC}_{\mathrm{uc}}$ refer to the accuracy of the changed class and the unchanged class respectively.

\begin{tabular}{ccc}
\hline Dataset & $\mathrm{PCC}_{\mathrm{c}}(\%)$ & $\mathrm{PCC}_{\text {uc }}(\%)$ \\
\hline $\mathrm{A}$ & 89.35 & 99.98 \\
$\mathrm{~B}$ & 83.71 & 99.82 \\
$\mathrm{C}$ & 99.76 & 99.25 \\
$\mathrm{D}$ & 97.91 & 99.97 \\
\hline
\end{tabular}

Table 9 shows the accuracy of simple pixel classification using the parallel clustering strategy embedded in TCCFCM. The accuracy (PCC) is greater than 99\%, showing that the parallel TCCFCM clustering strategy is able to provide credible pseudo-label training samples for deep learning models. 

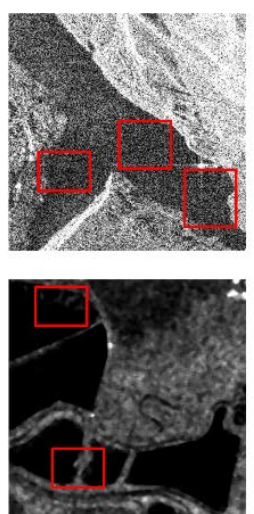

(a)
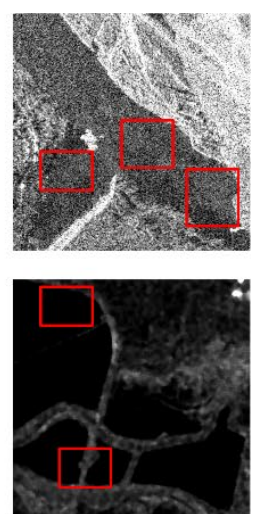

(b)
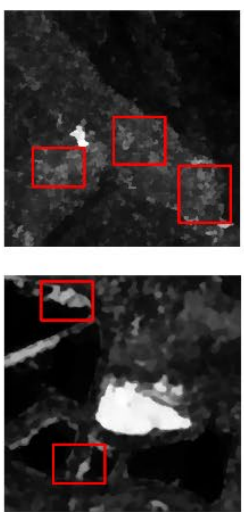

(c)
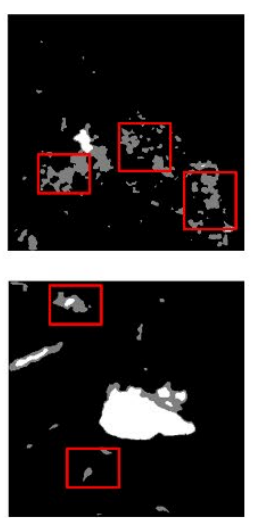

(d)
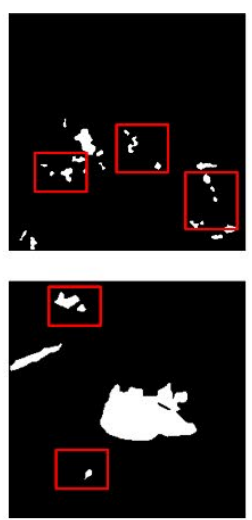

(e)
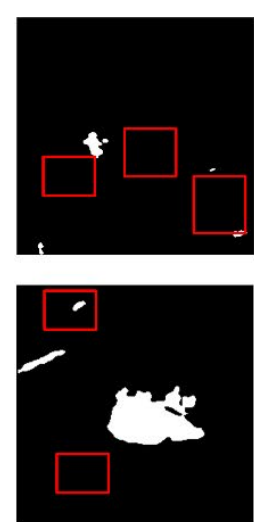

(f)
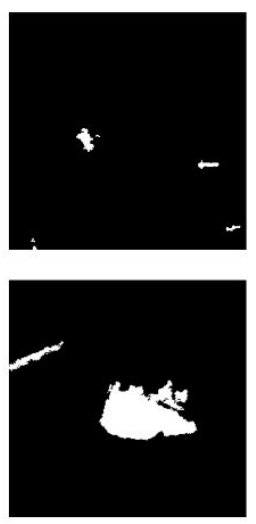

$(\mathrm{g})$

Fig. 14. Analysis of the difficulties in accurately detecting small area changes in dataset A and D. (a) and (b) are the original SAR images. (c) MSRDI. (d) Label map of parallel TCCFCM clustering; white pixels indicate simple pixels of the changed class, black pixels represent simple pixels of the unchanged class, grey pixels indicate hard pixels. (e) Change map by MTCCFCM. (f) Change map by RUSACD. (g) Ground reference map.

Two experiments were conducted on datasets A and D using deep learning (CWNN with DCGAN). In Fig. 14, the red windows indicate that many hard pixels are difficult to distinguish in MSRDI, which are classified accurately by the deep learning models. For Dataset D, there are some slight distortions between the bi-temporal SAR images, and they do not represent real land cover changes. RUSACD can distinguish well between the distortions and real changed pixels with the help of deep learning. In dataset A, the proposed RUSACD significantly increased the $\mathrm{KC}$ from $44.68 \%$ to $71.11 \%$, and $\mathrm{F}_{1}$ from $45.21 \%$ to $71.31 \%$ compared with MTCCFCM. This is further validated by the three other datasets, showing that CWNN with DCGAN can precisely classify hard pixels, thus, increasing change detection accuracy. By contrast, many false alarms are shown when classifying all pixels based on MSRDI and TCCFCM without the deep learning models.

The second experiment demonstrated the sample augmentation for small area change detection, where several data augmentation methods were used for comparative analysis, including simple linear generation (SLG) (Gao et al., 2019), ADASYN (He et al., 2008), and our DCGAN method. As shown in Fig. 15, DCGAN was the most effective augmentation method with the highest accuracy in $\mathrm{PCC}^{\mathrm{H}}$ and $\mathrm{F}_{1}$ score. The CWNN with sample augmentation can achieve greater accuracy than the benchmark on datasets B, C and D. Although the benchmark experiment results maintain a high accuracy on dataset $\mathrm{A}$, it depends on the distribution of hard pixels, which are mostly unchanged pixels in dataset A. The trained CWNN without sample augmentation failed to differentiate hard pixels, and classified them all as unchanged pixels. 


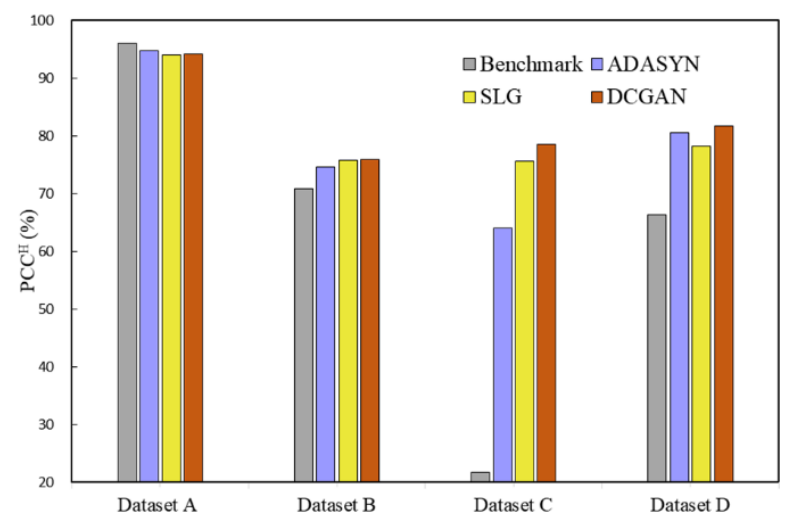

(a)

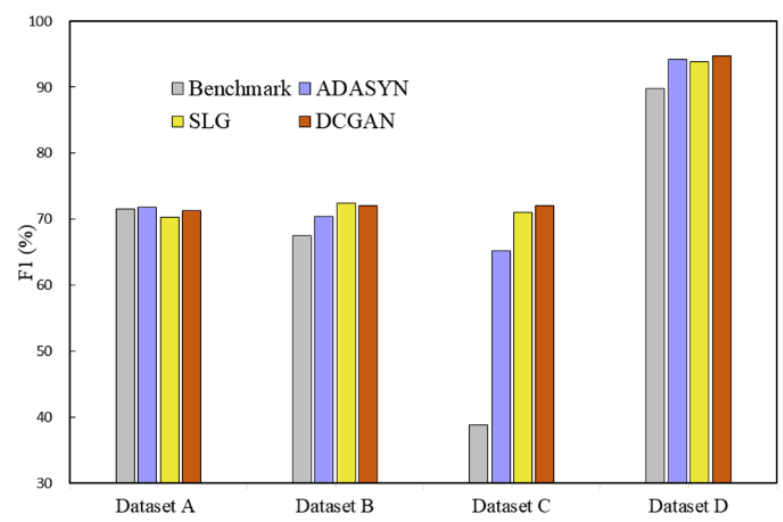

(b)
461

Fig. 15. Comparison of different sample augmentation methods. (a) The classification accuracy of hard pixels is the criterion. (b) The F1 score of the final change map is defined as the criterion.

\subsection{Ablation study}

We designed two ablation experiments to evaluate the robustness of hard pixel classification as a function of different network structures. The first experiment used generators with different numbers of convolution layers and convolution kernels to create the changed image patches. The network architectures of all DCGAN versions are listed in Table 10. Thereafter, the generated changed image patches and real pseudo-label image patches were used jointly to train the CWNN. The network structure of CWNN was fixed in the first experiment, with two datasets (B and D) adopted. The accuracy of hard pixel classification is listed in Table 11. In addition, the image patches generated by DCGAN with different network structures are shown in Fig. 14. $C_{i}$ represents the convolutional layer, where $i$ indexes the layer.

From Table 11, DCGAN ${ }^{3}$ shows advantages over the benchmark methods, achieving a $\mathrm{PCC}^{\mathrm{H}}$ of $80.82 \%$ on dataset $\mathrm{D}$. The patches generated by $\mathrm{DCGAN}^{1}$ with one-layer and a few convolution kernels were not beneficial to training a strong CWNN, since the simple network of DCGAN ${ }^{1}$ was unable to learn the visual features of the change classes effectively (Fig.16). In contrast, DCGAN ${ }^{4}$ and DCGAN 5 employed more layers and convolutional kernels, and resulted in learned features similar to the training data. For dataset B, the CWNN combined with DCGAN ${ }^{4}$ achieved the best performance, with a $\mathrm{PCC}^{\mathrm{H}}$ of $71.89 \%$, slightly higher than the $71.73 \%$ of DCGAN ${ }^{3}$. This is because dataset B presents a more complex background texture, which requires rich convolution kernels to learn detailed texture information. Taking all factors into account, DCGAN $^{3}$ was selected in the proposed RUSACD. The experimental results also demonstrated that a reasonable structure of DCGAN was useful to learn deep features of the changed class, where the generated 
samples include rich feature representations, thereby enhancing the discrimination capability of the trained 484 CWNN.

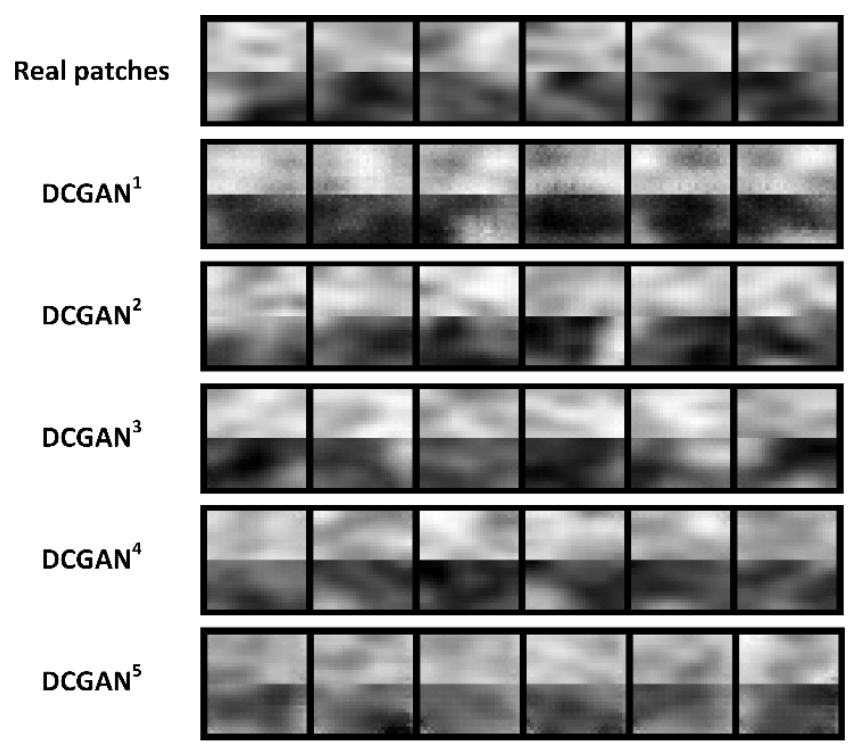

(a)
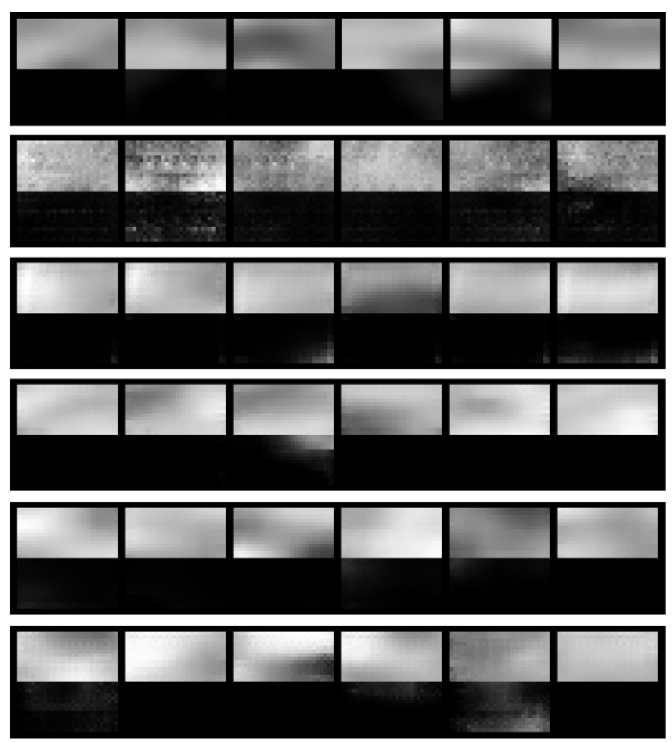

(b)

Fig.16. The changed image patches generated by DCGAN. (a) dataset B. (b) dataset D.

Table 10. The architecture of different versions of DCGANs. G and D refer to generator and discriminator respectively

\begin{tabular}{|c|c|c|c|c|c|c|c|c|c|}
\hline \multicolumn{2}{|c|}{ DCGAN $^{1}$} & \multicolumn{2}{|c|}{ DCGAN $^{2}$} & \multicolumn{2}{|c|}{ DCGAN $^{3}$} & \multicolumn{2}{|c|}{ DCGAN $^{4}$} & \multicolumn{2}{|c|}{ DCGAN $^{5}$} \\
\hline G & D & G & D & G & D & G & D & G & D \\
\hline \multirow[t]{5}{*}{$C_{1}: 8 \times 8 \times 64$} & $\mathrm{C}_{1}: 8 \times 8 \times 32$ & $C_{1}: 6 \times 6 \times 64$ & $C_{1}: 6 \times 6 \times 32$ & $\mathrm{C}_{1}: 4 \times 4 \times 64$ & $\mathrm{C}_{1}: 4 \times 4 \times 16$ & $C_{1}: 4 \times 4 \times 64$ & $\mathrm{C}_{1}: 4 \times 4 \times 8$ & $\mathrm{C}_{1}: 4 \times 4 \times 64$ & $\mathrm{C}_{1}: 4 \times 4 \times 4$ \\
\hline & & $\mathrm{C}_{2}: 4 \times 4 \times 32$ & $\mathrm{C}_{2}: 4 \times 4 \times 64$ & $\mathrm{C}_{2}: 4 \times 4 \times 32$ & $\mathrm{C}_{2}: 4 \times 4 \times 32$ & $\mathrm{C}_{2}: 4 \times 4 \times 32$ & $\mathrm{C}_{2}: 4 \times 4 \times 16$ & $\mathrm{C}_{2}: 3 \times 3 \times 32$ & $\mathrm{C}_{2}: 3 \times 3 \times 8$ \\
\hline & & & & $\mathrm{C}_{3}: 3 \times 3 \times 16$ & $\mathrm{C}_{3}: 3 \times 3 \times 64$ & $\mathrm{C}_{3}: 4 \times 4 \times 16$ & $\mathrm{C}_{3}: 4 \times 4 \times 32$ & $\mathrm{C}_{3}: 3 \times 3 \times 16$ & $\mathrm{C}_{3}: 3 \times 3 \times 16$ \\
\hline & & & & & & $\mathrm{C}_{4}: 3 \times 3 \times 8$ & $\mathrm{C}_{4}: 3 \times 3 \times 64$ & $\mathrm{C}_{4}: 3 \times 3 \times 8$ & $\mathrm{C}_{4}: 3 \times 3 \times 32$ \\
\hline & & & & & & & & $\mathrm{C}_{5}: 3 \times 3 \times 4$ & $\mathrm{C}_{5}: 3 \times 3 \times 64$ \\
\hline
\end{tabular}

Table 11. The PCC (\%) of hard pixel classification using DCGANs with different architectures for data augmentation.

\begin{tabular}{cccccc}
\hline & DCGAN $^{1}$ & DCGAN $^{2}$ & DCGAN $^{3}$ & DCGAN $^{4}$ & DCGAN $^{5}$ \\
\hline Dataset B & 70.51 & 70.64 & 71.73 & $\mathbf{7 1 . 8 9}$ & 71.84 \\
Dataset D & 74.95 & 74.25 & $\mathbf{8 0 . 8 2}$ & 77.74 & 74.22 \\
\hline
\end{tabular}

The second experiment used CWNNs with different numbers of layers and convolution kernels to 492 classify hard pixels, and the results are shown in Table 12, with the fixed DCGAN ${ }^{3}$ being used. The CWNN 3 493 acquired the highest accuracy (average $\mathrm{PCC}^{\mathrm{H}}$ of $78.56 \%$ ). The accuracy of shallow and deep CWNNs were

494 limited according to the $\mathrm{PCC}^{\mathrm{H}}$. CWNNs with small numbers of layers or convolution kernels tend to under-

495 fit because they are oversimplified models with insufficient learning capacity. On the contrary, CWNNs with 496 a large number of convolution kernels or layers tended to overfit through training, resulting in a decrease in 
classification accuracy. Based on the above experimental results, $\mathrm{CWNN}^{3}$ is demonstrated to be the most

suitable network architecture with a powerful discriminative capability.

Table 12. The PCC (\%) of hard pixel classification using CWNN with different architectures. The best results are shown

\begin{tabular}{|c|c|c|c|c|c|c|}
\hline \multirow{9}{*}{ Architecture } & $\mathrm{CWNN}^{1}$ & $\mathrm{CWNN}^{2}$ & $\mathrm{CWNN}^{3}$ & $\mathrm{CWNN}^{4}$ & $\mathrm{CWNN}^{5}$ & $\mathrm{CWNN}^{6}$ \\
\hline & \multirow{8}{*}{$\begin{array}{c}\mathrm{C}_{1}: 5 \times 5 \times 6 \\
\text { Pooling: } 2 \\
\text { FC: } 864\end{array}$} & \multirow{8}{*}{ 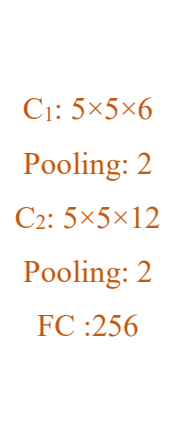 } & & $C_{1}: 5 \times 5 \times 6$ & & \\
\hline & & & $\mathrm{C}_{1}: 5 \times 5 \times 6$ & Pooling: 2 & $\mathrm{C}_{1}: 5 \times 5 \times 2$ & $\mathrm{C}_{1}: 5 \times 5 \times 18$ \\
\hline & & & Pooling: 2 & $\mathrm{C}_{2}: 3 \times 3 \times 12$ & Pooling: 2 & Pooling: 2 \\
\hline & & & $\mathrm{C}_{2}: 5 \times 5 \times 12$ & Pooling: 2 & $C_{2}: 5 \times 5 \times 4$ & $\mathrm{C}_{2}: 5 \times 5 \times 36$ \\
\hline & & & Pooling: 2 & $\mathrm{C}_{3}: 2 \times 2 \times 48$ & Pooling: 2 & Pooling: 2 \\
\hline & & & $\mathrm{C}_{3}: 4 \times 4 \times 96$ & Pooling: 2 & $\mathrm{C}_{3}: 4 \times 4 \times 32$ & $C_{3}: 4 \times 4 \times 288$ \\
\hline & & & FC: 96 & $\mathrm{C}_{4}: 2 \times 2 \times 96$ & FC: 32 & FC: 288 \\
\hline & & & & FC: 96 & & \\
\hline Dataset A & 86.33 & 96.63 & 92.98 & 94.88 & 97.96 & 95.12 \\
\hline Dataset B & 49.54 & 67.39 & 71.73 & 54.09 & 65.64 & 62.01 \\
\hline Dataset C & 68.37 & 64.50 & 68.69 & 65.52 & 64.51 & 70.12 \\
\hline Dataset D & 71.56 & 77.90 & 80.82 & 70.52 & 70.16 & 68.60 \\
\hline Average & 68.95 & 76.61 & 78.56 & 71.25 & 74.57 & 73.96 \\
\hline
\end{tabular}

\subsection{Parameter analysis}

To test the sensitivity of the results to parameter choice, we investigated the effect of the constraint parameter $\beta$ in TCCFCM on change detection accuracy. We fixed other parameters, and assigned a set of values to $\beta$ as shown in Fig. 15. RUSACD was not sensitive to the parameter $\beta$ for all datasets, and the change detection accuracy was the highest (Fig. 15(b)). The MTCCFCM exhibited stable and satisfactory performance for datasets B, C and D (Fig. 15(a)). The accuracy for dataset A declined when $\beta$ was less than 0.4. These results indicate that RUSACD is robust and accurate compared with MTCCFCM for small area change detection.

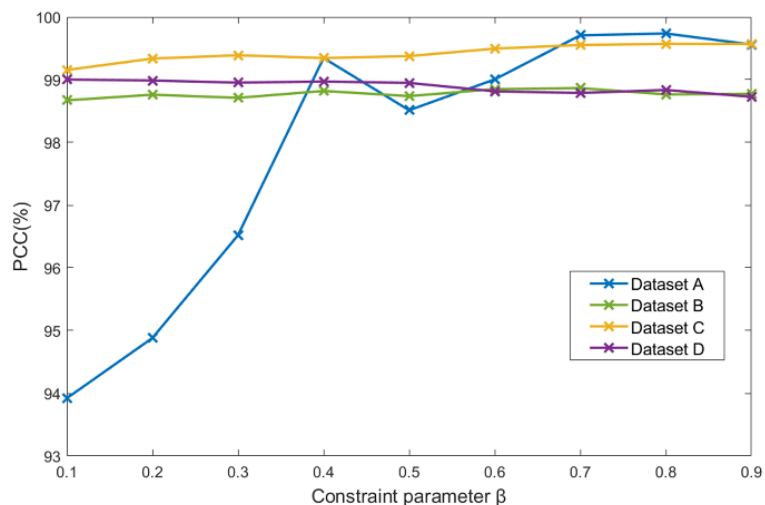

(a)

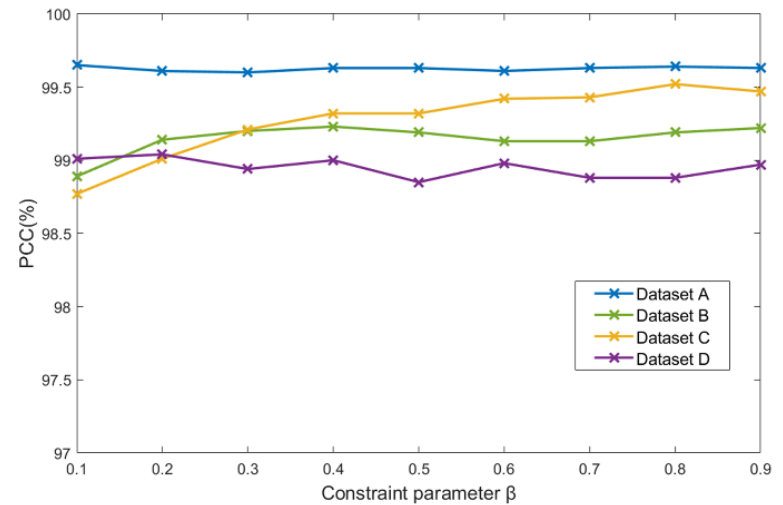

(b)

Fig. 17. The relationship between change detection accuracy and parameter $\beta$. (a) Results of MTCCFCM. (b) Results of 


\subsection{Discussion}

In multi-temporal SAR image change detection, the speckle noise and image distortion produced in the image acquisition process often cause false changes, leading to a decrease in change detection accuracy. For small area change detection, the number of false changed pixels may be similar to the number of real changed pixels, leading to the problems addressed in this paper. In this research, we proposed RUSACD, as a stepwise modular framework, and demonstrated that it was the most accurate method across a series of state-ofthe-art benchmarks for small area change detection.

In the DI generation module, the developed MSRDI aimed to increase the gap between unchanged and changed areas, as well as suppressing speckle noise. It was also able to reduce the interference caused by false-changed pixels.

In the clustering module in the DI analysis, existing FCM-based algorithms usually produce incorrect shifts of cluster prototypes when the number of changed pixels is far less than for unchanged pixels. The TCCFCM was proposed to maintain the appropriate direction of the clustering algorithm through the optimisation process by adopting preliminary centres as constraint terms in the objective function. Furthermore, instead of clustering into two categories, TCCFCM used a parallel clustering strategy, dividing the MSRDI into three classes, which was beneficial for discriminating accurately between false changed pixels and real changed pixels.

In the intermediate (hard) pixel classification module, the CWNN was employed for small area change detection. However, as is typical for small area change detection, there were too few training samples of the changed class to train the CWNN. To avoid an imbalance between the changed and unchanged training samples a DCGAN was applied to enrich the training samples of the minority class. Further, compared to other data augmentation methods, DCGAN explored the visual feature space through the learning of image patches, and captured effectively the greatest potential discriminative information for the changed class. The augmented data cover the feature space of the changed class, which supports the training of CWNN.

Rigorous evaluation was undertaken of each module in RUSACD by comparing against several benchmark approaches. The experimental results demonstrate the effectiveness and robustness of each module in RUSACD. The proposed method has the benefit that each module can be extended readily and, indeed, the entire framework can be developed as an end-to-end deep network. In future research, the method 
should be evaluated for a wider range of datasets and applications such as change detection using optical $541 \quad$ Sentinel-2 images.

\section{Conclusion}

In this paper, a modular RUSACD framework composed of MSRDI, TCCFCM, CWNN and DCGAN was proposed for small area change detection from multi-temporal SAR images. Experiments on four real SAR datasets demonstrated the effectiveness and robustness of each module of RUSACD for small area change detection. The MSRDI in the framework enhances the edges of changed areas and minimises speckle noise and background pixels, increasing the separability between changed and unchanged pixels. The TCCFCM avoids incorrect transfer of clustering prototypes caused by class imbalanced samples, and together with a parallel clustering strategy, the pseudo-label training samples are selected automatically in an unsupervised approach. CWNN obtains discriminative features from the two original SAR images and the MSRDI, while DCGAN further expands the pseudo-label training samples of the changed class, solving the problem of insufficient training samples. In addition, the adversarial learning process of DCGAN can capture effectively the potential visual features of the changed class, which supports the training of a strong classifier. We conclude that RUSACD offers many advantages for small area change detection and produced consistently the greatest accuracy of classification amongst the tested benchmarks.

\section{Acknowledgement}

This work was supported by the National Science Foundation of China (61301224) and the Chongqing Basic and Frontier Research Project (cstc2017jcyjA1378). The authors are grateful to the anonymous reviewers for their constructive comments which increased greatly the quality of this manuscript.

\section{Declaration of Competing Inerest}

The authors declare that they have no conflicts of interest to disclose.

\section{Reference}

Achanta, R., Shaji, A., Smith, K., Lucchi, A., Fua, P., Susstrunk, S., 2012. SLIC superpixels compared to state-of-the-art superpixel methods. IEEE Trans. Pattern Anal. Mach. Intell. 34(11), 2274-2282. https://doi.org/10.1109/TPAMI.2012.120.

Brunner, D., Lemoine, G., Bruzzone, L., 2010. Earthquake damage assessment of buildings using VHR optical and SAR imagery. IEEE Trans. Geosci. Remote Sens. 48(5), 2403-2420. https://doi.org/10.1109/ tgrs.2009.2038274. 
Cao, S., Du, M., Zhao, W., Hu, Y., Mo, Y., Chen, S., Cai, Y., Peng, P., Zhang, C., 2020. Multi-level monitoring of threedimensional building changes for megacities: Trajectory, morphology, and landscape. ISPRS J. Photogramm. Remote Sens. 167, 54-70. https://doi.org/10.1016/j.isprsiprs.2020.06.020.

Celik, T., 2009. Unsupervised change detection in satellite images using principal component analysis and k-means clustering. IEEE Geosci. Remote Sens. Lett. 6(4), 772-776. https://doi.org/10.1109/LGRS.2009.2025059.

Cozzolino, D., Verdoliva, L., Scarpa, G., Poggi, G., 2020. Nonlocal CNN SAR image despeckling. Remote Sens. 17(6), 1097-1101. https://doi.org/10.3390/rs12061006.

Deledalle, C-A., Denis, L., Tupin, F., Reigber, A., Jager, M., 2015. NL-SAR: A unified nonlocal framework for resolutionpreserving (Pol) (In) SAR denoising. IEEE Trans. Geosci. Remote Sens. 53(4), 2021-2038. https://doi.org/10.1109/TGRS.2014.2352555.

Duan, Y., Liu, F., Jiao, L., Zhao, P., Zhang, L., 2017. SAR Image segmentation based on convolutional-wavelet neural network and markov random field. Pattern Recognit. 64, 255-267. https://doi.org/10.1016/ j.patcog.2016.11.015.

Frid-Adar, M., Diamant, I., Klang, E., Amitai, M., Goldberger, J., Greenspan, H., 2018. GAN-based synthetic medical image augmentation for increased CNN performance in liver lesion classification. Neurocomputing. 321, 321-331. https://doi.org/10.1016/j.neucom.2018.09.013.

Gao, F., Dong, J., Li, B., Xu, Q., 2016. Automatic change detection in synthetic aperture radar images based on PCANet. IEEE Geosci. Remote Sens. Lett. 13(12), 1792-1796. https://doi.org/10.1109/LGRS.2016.2611001.

Gao, F., Dong, J., Li. B., Xu, Q., Xie, C., 2016. Change detection from synthetic aperture radar images based on neighborhood-based ratio and extreme learning machine. J. Appl. Remote Sens, 10(4), 046019. https://doi.org/ 10.1117/1.jrs.10.046019.

Gao, F., Wang, X., Gao, Y., Dong, J., Wang, S., 2019. Sea ice change detection in SAR images based on convolutionalwavelet neural networks. IEEE Geosci. Remote Sens. Lett. 16(8), 1240-1244. https://doi.org/ 10.1109/LGRS.2019.2895656.

Geng, J., Ma, X., Zhou, X., Wang, H., 2019. Saliency-guided deep neural networks for SAR image change detection. IEEE Trans. Geosci. Remote Sens. 57(10), 7365-7377. https://doi.org/10.1109/TGRS.2019.2913095.

Goodfellow, I., Pouget-Abadie, J., Mirza, M., Xu, B., Warde-Farley, D., Ozair, S.,Courville, A., Bengio, Y., 2014. Generative adversarial nets. Proc. NIPS. 2672-2680. https://arxiv.org/pdf/1406.2661.

Gong, M., Cao, Y., Wu, Q., 2012. A neighborhood-based ratio approach for change detection in SAR images. IEEE Geosci. Remote Sens. Lett. 9(2), 307-311. https://doi.org/10.1109/LGRS.2011.2167211.

Gong, M., Yang, H., Zhang, P., 2017. Feature learning and change feature classification based on deep learning for ternary change detection in SAR images. ISPRS J. Photogramm. Remote Sens. 129, 212-225. https://doi.org/10.1016/j.isprsiprs.2017.05.001.

Gong, M., Zhao, J., Liu, J., Miao, Q., Jiao, L., 2016. Change detection in synthetic aperture radar images based on deep neural networks. IEEE Trans. Neural Netw. Learn. Syst, 27(1), 125-138. https://doi.org/10.1109/ tnnls.2015.2435783.

Gong, M., Zhou, Z., Ma, J., 2012. Change detection in synthetic aperture radar images based on image fusion and fuzzy clustering. IEEE Trans. Image Process. 21(4), 2141-2151. https://doi.org/10.1109/TIP.2011.2170702. 
He, H., Bai, Y., Garcia, E, A., Li, S., 2008. ADASYN: Adaptive synthetic sampling approach for imbalanced learning. Proc. Int'1 Joint Conf. Neural Netw. 1322-1328. https://doi.org/10.1109/ijenn.2008.4633969.

Huang, S., Cai, X., Chen, S., Liu, D., 2011. Change detection method based on fractal model and wavelet transform for multitemporal SAR images. Int. J. Appl. Earth Obs. Geoinf. 13(6), 863-872. https://doi.org/10.1016/ j.jag.2011.05.018. Inglada, J., Mercier, G., 2007. A new statistical similarity measure for change detection in multitemporal SAR images and its extension to multiscale change analysis. IEEE Trans. Geosci. Remote Sens. 45(5), 1432-1445. https://doi.org/10.1109/TGRS.2007.893568.

Jia, L., Li, M., Zhang, P., Wu, Y., Zhu, H., 2016. SAR image change detection based on multiple kernel k-means clustering with local-neighborhood information. IEEE Geosci. Remote Sens. Lett. 13(6), 856-860. https://doi.org/10.1109/lgrs.2016.2550666.

Kalaiselvi, S., Gomathi, V., 2020. $\alpha$-cut induced fuzzy deep neural network for change detection of SAR images. Appl. Soft Comput. 95, 106510. https://doi.org/10.1016/j.asoc.2020.106510.

Kim, Y., Lee, M, J., 2020. Rapid change detection of flood affected area after collapse of the Laos Xe-Plain Xe-Namnoy dam using Sentinel GRD data. Remote Sens. 12(12), 1978. https://doi.org/10.3390/rs12121978.

Krinidis, S., Chatzis, V., 2010. A robust fuzzy local information c-means clustering algorithm. IEEE Trans. Image Process. 19(5), 1328-1337. https://doi.org/10.1109/TIP.2010.2040763.

Lei, Y., Liu, X., Shi, J., Lei, C., Wang, J., 2019. Multiscale superpixel segmentation with deep features for change detection. IEEE Access. 7, 36600-36616. https://doi.org/10.1109/ACCESS.2019.2902613.

Li, H., Gong, M., Wang, C., Miao, Q., 2018. Self-paced stacked denoising autoencoders based on differential evolution for change detection. Appl. Soft Comput. 71, 698-714. https://doi.org/10.1016/j.asoc.2018.07.021.

Li, H, C., Celik, T., Longbotham, N., Emery, W, J., 2015. Gabor feature based unsupervised change detection of multitemporal SAR images based on two-level clustering. IEEE Geosci. Remote Sens. Lett. 12(12), 2458-2462. https://doi.org/10.1109/LGRS.2015.2484220.

Li, H, C., Yang, G., Yang, W., Du, Q., Emery, W. J., 2020. Deep nonsmooth nonnegative matrix factorization network factorization network with semi-supervised learning for SAR image change detection. ISPRS J. Photogramm. Remote Sens. 160, 167-179. https://doi.org/10.1016/j.isprsjprs.2019.12.002.

Li, L., Wang, C., Zhang, H., Zhang, B., Wu, F. 2019. Urban building change detection in SAR images using combined differential image and residual U-Net network. Remote Sens. 11(9). 1091. https://doi.org/ 10.3390/rs11091091.

Li, M., Li, M., Zhang, P., Wu, Y., Song, W., An, L., 2019. SAR image change detection using PCANet guided by saliency detecion. IEEE Geosci. Remote Sens. Lett. 16(3), 402-406. https://doi.org/10.1109/LGRS.2018.2876616.

Li, Y., Peng, C., Chen, Y., Jiao, L., Zhou, L., Shang, R., 2019. A deep learning method for change detection in synthetic aperture radar images. IEEE Trans. Geosci. Remote Sens. 57(8), 5751-5763. https://doi.org/10.1109/ TGRS.2019.2901945.

Pantze, A., Santoro, M., Fransson, J. E. S. 2014. Change detection of boreal forest using bi-temporal ALOS PALSAR backscatter data. Remote Sens. Environ. 155, 120-128. https://doi.org/10.1016/j.rse.2013.08.050.

Radford, A., Metz, L., Chintala, S., 2015. Unsupervised representation learning with deep convolutional generative adversarial networks. arXiv preprint arXiv:1511.06434. https://arxiv.org/pdf/1511.06434.pdf. 
Saha, S., Bovolo, F., Bruzzone, L., 2020. Building change detection in VHR SAR images via unsupervised deep tanscoding.

Shorten, C., Khoshgoftaar, T. M., 2019. A survey on image data augmentation for deep learning. J. Big Data, 6(1). https://doi.org/10.1186/s40537-019-0197-0.

Sun, Z., Zhang, Z., Chen, Y., Liu, S., Song, Y., 2020. Frost filtering algorithm of SAR images with adaptive windowing and adaptive tuning factor. IEEE Geosci. Remote Sens. Lett. 17(6), 1097-1101. https://doi.org/10.1109/LGRS.2019.2939208.

Tian, D., Gong, M., 2018. A novel edge-weight based fuzzy clustering method for change detection in SAR images. Inf. Sci. 467, 415-430. https://doi.org/10.1016/j.ins.2018.08.015.

Wang, J., Yang, X., Yang, X. Jia, L., Fang, S., 2020. Unsupervised change detection between SAR images based on hypergraphs. ISPRS J. Photogramm. Remote Sens. 164, 61-72. https://doi.org/10.1016/j.isprsjprs.2020.04.007.

Wang, S., Jiao, L., Yang, S., 2016. SAR Images Change detection based on spatial coding and nonlocal similarity pooling. IEEE J. Sel. Top. Appl. Earth Observ. Remote Sens. 9(8), 3452-3466. https://doi.org/10.1109/jstars.2016.2547638.

Zheng, Y., Jiao, L., Liu, H., Zhang, X., Hou, B., Wang, S., 2017. Unsupervised saliency-guided SAR image change detection. Pattern Recognit, 61, 309-326. https://doi.org/10.1016/j.patcog.2016.07.040.

Zheng, Z., Zhang, X., Hou, B., Liu, G., 2014. Using combined difference image and k-means clustering for SAR image change detection. IEEE Geosci. Remote Sens. Lett. 11(3), 691-695. https://doi.org/10.1109/LGRS.2013.2275738.

Zhuang, H., Fan, H., Deng, K., Yao, G., 2018. A spatial-temporal adaptive neighborhood-based ratio approach for change detection in SAR images. Remote Sens. 10(8), 1295. https://doi.org/10.3390/rs10081295.

Zhuang, H., Tan, Z., Deng, K., Yao, G., 2020. Adaptive generalized likelihood ratio test for change detection in SAR image. IEEE Geosci. Remote Sens. Lett. 17(3), 416-420. https://doi.org/10.1109/LGRS.2019.2922198. 